\title{
DYNAMIQUE DES HOMÉOMORPHISMES DU PLAN AU VOISINAGE D'UN POINT FIXE
}

\author{
PAR PATRICE LE CALVEZ
}

RÉSUMÉ. - Nous étudions la dynamique d'un homéomorphisme d'une surface au voisinage d'un point fixe et cherchons à calculer la suite des indices de Lefschetz des itérés en ce point. Nous en déduisons l'existence d'une infinité d'orbites périodiques pour certains homéomorphismes conservatifs de surfaces.

(C) 2003 Éditions scientifiques et médicales Elsevier SAS

ABSTRACT. - We study the dynamics of a homeomorphism of a surface near a fixed point. We compute the sequence of the Lefschetz indices of the iterates of the map. We deduce the existence of an infinite number of periodic orbits for some conservative homeomorphisms of surfaces.

(C) 2003 Éditions scientifiques et médicales Elsevier SAS

L'objet de cet article est l'étude des homéomorphismes de surfaces au voisinage d'un point fixe dans le but de montrer l'existence d'infinité d'orbites périodiques pour certains homéomorphismes conservatifs. Plus précisément nous montrerons les deux résultats suivants.

THÉORÈME 1. - Si $f: S^{2} \rightarrow S^{2}$ est un homéomorphisme qui a au moins trois points fixes et qui n'a pas de point errant, alors $f$ a une infinité d'orbites périodiques.

THÉORÈme 2. - Soit $f$ un homéomorphisme du tore $\mathbf{T}^{2}=\mathbf{R}^{2} / \mathbf{Z}^{2}$ homotope à l'identité et $\tilde{f}$ un relèvement de $f$ à $\mathbf{R}^{2}$. On suppose que f préserve une mesure borélienne de probabilité $\mu$ chargeant les ouverts et ayant un vecteur de rotation

$$
\rho(\mu)=\int_{\mathbf{T}^{2}} \tilde{f}-I d d \mu
$$

égal à 0 . Alors $f$ a une infinité d'orbites périodiques.

Le premier résultat était déja connu pour les homéomorphismes topologiquement transitifs (voir M. Handel [18]) et pour les homéomorphismes qui préservent une mesure de probabilité chargeant les ouverts (voir J. Franks [16]).

La formule de Lefschetz est un outil majeur dans la preuve de ces résultats. Ainsi, les problèmes locaux qui nous intéresseront seront les suivants :

- si $z \in \mathbf{R}^{2}$ est un point fixe d'un homéomorphisme (non nécessairement conservatif) localement défini au voisinage de $z$, que peut-on dire de la suite $\left(i\left(f^{k}, z\right)\right)_{k \geqslant 1}$ (quand elle est définie) des indices de Lefschetz des itérés de $f$ ?

- quel est le lien entre la structure de cette suite et la dynamique de $f$ au voisinage de 0 ?

Il y a des cas où la suite des indices de Lefschetz des itérés est déja connue, c'est le cas où le point fixe est accumulé par des orbites périodiques : la suite est alors constante égale à 1 . Ceci 
nous amène à faire une première classification entre les points fixes accumulés (par des orbites périodiques) et ceux qui ne le sont pas. Nous porterons une plus grande attention à ces derniers.

Un autre cas, celui-ci très simple, où la suite est connue et également constante égale à 1 est le cas où le point fixe admet un système de voisinages, formé de domaines attractifs ou répulsifs dont la frontière est une courbe de Jordan. Cette situation, qualifiée de dissipative, est bien sûr impossible pour un homéomorphisme sans point errant.

La suite $\left(i\left(f^{k}, z\right)\right)_{k \geqslant 1}$ a été étudiée avec J.-C. Yoccoz (voir [24]) dans le cas où le point fixe $z$ est localement maximal, c'est-à-dire dans le cas où il existe un voisinage $U$ tel que $z$ est le seul point d'orbite totale contenue dans $U$, et où $z$ n'est pas de type dissipatif. La suite est alors périodique. Plus précisément, il existe des entiers $q \geqslant 1$ et $r \geqslant 1$ tels que pour tout $k \geqslant 1$, on a :

$$
\begin{cases}i\left(f^{k}, z\right)=1 & \text { si } k \notin q \mathbf{Z}, \\ i\left(f^{k}, z\right)=1-r q & \text { si } k \in q \mathbf{Z} .\end{cases}
$$

Ce résultat a été généralisé (voir [25]) dans le cas où $z$ n'est pas dissipatif et où il existe un voisinage simplement connexe $U$ ne contenant aucune orbite périodique autre que $\{z\}$ et tel que

$$
\bigcap_{k \in \mathbf{Z}} f^{-k}(\bar{U}) \subset U
$$

Un point fixe admettant un système fondamental de voisinages simplement connexes $U$ vérifiant

$$
\bigcap_{k \in \mathbf{Z}} f^{-k}(\bar{U}) \subset U
$$

sera qualifié de point-selle.

Les points qui nous intéresseront particulièrement sont les points fixes non accumulés qui ne sont ni dissipatifs, ni des points-selles. Un exemple naturel est celui d'un point fixe indifférent d'une application holomorphe univalente (voir R. Perez-Marco [30]). Nous verrons comment la théorie des bouts premiers permet de ramener cette situation à celle des points dissipatifs et des points-selles.

Cette étude nous fera retrouver ou améliorer certains résultats locaux des homéomorphismes du plan que nous utiliserons alors dans la démonstration des théorèmes 1 et 2.

Nous montrerons le résultat suivant, dû à S. Pelikan et E. Slaminka [31] (et aux arguments précis de M. Bonino [3]), et montré auparavant pour les difféomorphismes par N.A. Nikishin [29] et C.P. Simon [34].

THÉORÈme 3. - Si $f: W \rightarrow W^{\prime}$ est un homéomorphisme entre deux voisinages de 0 dans $\mathbf{R}^{2}$ qui préserve une mesure borélienne chargeant les ouverts et qui admet 0 comme unique point fixe, alors $i(f, 0) \leqslant 1$.

Plus précisément nous montrerons :

THÉORÈME 4. - Soit $f: W \rightarrow W^{\prime}$ un homéomorphisme entre deux voisinages de 0 dans $\mathbf{R}^{2}$ qui admet 0 comme unique point fixe, et tel que $i(f, 0) \geqslant 2$. On a alors les deux propriétés suivantes:

(i) il existe un domaine $V \subset W$ tel que la suite $\left(f^{n}(V)\right)_{n \geqslant 0}$ est bien définie et formée de domaines disjoints deux à deux de $W$;

(ii) il existe un domaine $V^{\prime} \subset W^{\prime}$ tel que la suite $\left(f^{n}\left(V^{\prime}\right)\right)_{n \leqslant 0}$ est bien définie et formée de domaines disjoints deux à deux de $W^{\prime}$. 
Nous retrouverons ensuite le résultat de M. Brown [6] suivant :

THÉORÈME 5. - Soit $f: W \rightarrow W^{\prime}$ un homéomorphisme entre deux voisinages de 0 dans $\mathbf{R}^{2}$ qui admet 0 comme unique point fixe, et tel que $i(f, 0) \neq 1$, alors l'indice $i\left(f^{k}, 0\right)$ est bien défini pour tout $k \neq 0$ et indépendant de $k$.

On précisera ce résultat :

THÉORÈME 6. - Soit $f: W \rightarrow W^{\prime}$ un homéomorphisme entre deux voisinages de 0 dans $\mathbf{R}^{2}$ qui fixe 0 . Si la suite $\left(i\left(f^{k}, 0\right)\right)_{k \in \mathbf{Z}}$ est bien définie, il existe $q \geqslant 1$ et $r \in \mathbf{Z}$ tels que pour tout $k \geqslant 1$ :

$$
\begin{cases}i\left(f^{k}, 0\right)=1 & \text { si } k \notin q \mathbf{Z}, \\ i\left(f^{k}, 0\right)=r & \text { si } k \in q \mathbf{Z} .\end{cases}
$$

Une approche différente de l'étude locale des homéomorphismes au voisinage d'un point fixe a permis également à $\mathrm{F}$. Le Roux [26] de retrouver les résultats précédents.

Les neuf premiers paragraphes de l'article portent sur la dynamique locale, les trois derniers sur la dynamique globale.

Au paragraphe 1 nous introduisons les premières définitions et énonçons quelques lemmes fréquemment utilisés par la suite.

Au paragraphe 2, nous donnons deux classifications distinctes des points fixes, en particulier nous introduisons précisément la notion de point indifférent, de point dissipatif et de point-selle.

Au paragraphe 3, nous rappelons ce qui est connu sur la suite des indices de Lefchetz en un point fixe pour les itérés d'un homéomorphisme.

Aux paragraphes 4 et 5 nous rappelons certains résultats de la théorie des bouts premiers, en particulier le lien entre la dynamique et les bouts premiers.

Les paragraphes 6,7 et 8 , consacrés à l'étude des points indifférents non accumulés, sont les paragraphes principaux. Nous définissons le nombre de rotation d'un tel point, puis nous introduisons la notion d'éclatement. Nous montrons alors comment construire, à partir d'un éclatement convenablement choisi, un homéomorphisme dont tous les points périodiques sont dissipatifs ou de type selle et comment calculer les indices de Lefschetz des itérés au point fixe initial en utilisant les résultats de [25] sur les nouveaux points périodiques.

Nous prouvons les théorèmes $3,4,5$ et 6 au paragraphe 9 .

Nous débutons l'étude globale des homéomorphismes au paragraphe 10, où nous rappelons quelques résultats sur les homéomorphismes conservatifs de l'anneau. Nous utilisons ces résultats globaux ainsi que les résultats locaux montrés auparavant pour donner une preuve du théorème 1 au paragraphe 11 , du théorème 2 au paragraphe 12 .

\section{Rappels, notations}

\subsection{Domaines de Jordan}

On rappelle qu'une courbe de Jordan est une partie du plan euclidien homéomorphe au cercle unité $S^{1}$ et qu'un domaine de Jordan est la composante connexe bornée du complémentaire d'une courbe de Jordan. Tout domaine de Jordan est homéomorphe à la boule unité $\mathbf{D}$ et son adhérence à $\overline{\mathbf{D}}$. Si on oriente le plan $\mathbf{R}^{2}$, on a une orientation naturelle sur toute courbe de Jordan. 


\subsection{Indices}

Soit $f: W \rightarrow W^{\prime}$ un homéomorphisme entre deux parties ouvertes de $\mathbf{R}^{2}$ et Fix $(f)$ l'ensemble des points fixes de $f$.

Si $C$ est une courbe de Jordan contenue dans $W$ ne rencontrant pas $\operatorname{Fix}(f)$, on appelle indice de $f$ le long de $C$, le degré $i(f, C)$ de l'application

$$
\begin{aligned}
\chi: S^{1} & \rightarrow S^{1}, \\
t & \mapsto \frac{f(\gamma(t))-\gamma(t)}{\|f(\gamma(t))-\gamma(t)\|},
\end{aligned}
$$

où $\gamma: S^{1} \rightarrow C$ est une paramétrisation préservant l'orientation (l'indice est indépendant de l'orientation choisie sur $\mathbf{R}^{2}$ ).

Si $z$ est un point fixe isolé de $f$, et si $U$ est un domaine de Jordan qui est un voisinage suffisamment petit de $z$, alors l'entier $i(f, \partial U)$ est bien défini et indépendant de $U$ : c'est par définition l'indice de Lefschetz de $z$, on le note $i(f, z)$.

Si $U \subset W$ est un domaine de Jordan ayant un nombre fini de points fixes et si sa frontière est contenue dans $W$ et ne rencontre pas $\operatorname{Fix}(f)$, on a

$$
i(f, \partial U)=\sum_{z \in \operatorname{Fix}(f) \cap U} i(f, z) .
$$

L'indice $i(f, z)$ ne dépend que du germe de $f$ en 0 , plus précisément de la classe de conjugaison de ce germe. On peut donc définir l'indice de Lefschetz d'un point fixe isolé d'un homéomorphisme d'une surface.

\subsection{Continus}

On rappelle qu'un continu est une partie compacte et connexe d'un espace topologique. On dira qu'un continu $K$ d'une surface $M$ est plein si son complémentaire est connexe et qu'il est contractile s'il est contenu dans un domaine simplement connexe de $M$. Si $K$ est plein et contractile, on obtient une nouvelle surface $\widehat{M}$ homéomorphe à $M$ en identifiant $K$ à un point $\{K\}$.

Si $K \subset M$ est un continu plein contractile invariant par un homéomorphisme $f: W \rightarrow W^{\prime}$ entre deux voisinages ouverts de $K$ dans $M$, on obtient naturellement un homéomorphisme $\hat{f}: \widehat{W} \rightarrow \widehat{W}^{\prime}$ entre deux voisinages ouverts du point fixe $\{K\}$ dans $\widehat{M}$. S'il existe un voisinage $U \subset W$ de $K$ tel que $f$ n'a pas de point fixe dans $U \backslash K$, le point $\{K\}$ est un point fixe isolé de $\hat{f}$ et on peut définir l'indice

$$
i(f, K)=i(\hat{f},\{K\}) .
$$

Si $f$ n'a qu'un nombre fini de points fixes dans $K$, on a

$$
i(f, K)=\sum_{z \in \operatorname{Fix}(f) \cap K} i(f, z) .
$$

\subsection{Lemme de Brouwer}

On utilisera souvent le résultat suivant, dû à L.E.J. Brouwer [4] (voir également [5,10] ou [17]).

$4^{\mathrm{e}}$ SÉRIE - TOME $36-2003-\mathrm{N}^{\circ} 1$ 
Proposition 1.1. - Soit $f: \mathbf{R}^{2} \rightarrow \mathbf{R}^{2}$ un homéomorphisme préservant l'orientation. Si $f$ a un point non errant qui n'est pas un point fixe (par exemple un point périodique de période $q \geqslant 2$ ), alors il existe une courbe de Jordan $C$, disjointe de $\operatorname{Fix}(f)$, telle que $i(f, C)=1$.

\subsection{Homéomorphismes semi-stables}

Notons

$$
\begin{aligned}
\Pi: \mathbf{R} & \rightarrow S^{1}, \\
\theta & \mapsto(\cos \theta, \sin \theta)
\end{aligned}
$$

le revêtement universel de $S^{1}$. On dira qu'un homéomorphisme préservant l'orientation $\varphi$ de $S^{1}$ est semi-stable à droite (resp. semi-stable à gauche) si, étant donné un relèvement $\tilde{\varphi}$ de $\varphi$ à $\mathbf{R}$, il existe $p \in \mathbf{Z}$ et $q>0$ tels que la fonction $\tilde{\varphi}^{q}-2 p \pi$ s'annule et garde un signe positif ou nul (resp. négatif ou nul). Plus concrètement un homéomorphisme est semi-stable à droite (resp. à gauche) si son nombre de rotation $p / q+\mathbf{Z}$ est rationnel et si aucun point n'est envoyé "à gauche" (resp. "à droite") par $\varphi^{q}$. Les homéomorphismes semi-stables à la fois à droite et à gauche sont les homéomorphismes périodiques.

\subsection{Ensembles isolants}

Soit $f: W \rightarrow W^{\prime}$ un homéomorphisme entre deux parties ouvertes $W$ et $W^{\prime}$ d'une surface. On dit qu'un ensemble ouvert $V$, d'adhérence contenue dans $W$, est isolant si on a

$$
\bigcap_{k \in \mathbf{Z}} f^{-k}(\bar{V}) \subset V
$$

\subsection{Un lemme de connexité}

Nous utiliserons souvent le lemme suivant (voir par exemple [20], p. 47).

LEMME 1.2. - Soit $X$ un espace topologique compact et connexe et $Y$ une partie fermée de $X$. Alors l'adhérence de toute composante connexe de $X \backslash Y$ rencontre $Y$.

\section{Classifications des points fixes}

Nous donnerons dans ce paragraphe deux classifications des points fixes d'un homéomorphisme local du plan. Nous supposerons toujours que les homéomorphismes préservent l'orientation.

\subsection{Points accumulés et non accumulés}

Soit $f: W \rightarrow W^{\prime}$ un homéomorphisme entre deux voisinages $W$ et $W^{\prime}$ de 0 dans $\mathbf{R}^{2}$, préservant l'orientation et laissant fixe 0 . On dira que 0 est accumulé si tout voisinage de 0 inclus dans $W$ contient une orbite périodique autre que 0 , on dira qu'il est non accumulé dans le cas contraire. Dans le cas particulier où il existe $q \geqslant 1$ tel que tout voisinage de 0 inclus dans $W$ contient une orbite périodique de période $q$ autre que 0 , on dira que 0 est dégénéré. On a donc une première classification entre les points fixes non accumulés, les points fixes accumulés non dégénérés et les points fixes dégénérés. Cette classification ne dépend que du germe de $f$ en 0 et plus précisément de la classe de conjugaison de ce germe. 


\subsection{Points indifférents}

On rappelle que si $f: W \rightarrow W^{\prime}$ est une application holomorphe univalente entre deux voisinages $W$ et $W^{\prime}$ de 0 dans $\mathbf{C}$, le point 0 est indifférent si $\left|f^{\prime}(0)\right|=1$. Un tel point vérifie la propriété suivante, montrée par R. Perez-Marco [30] :

Si $U$ est un domaine de Jordan contenant 0 et d'adhérence contenue dans $W$, alors la composante connexe $K$ de l'ensemble $\bigcap_{k \in \mathbf{Z}} f^{-k}(\bar{U})$ qui contient 0 rencontre $\partial U$.

On va généraliser cette notion. Si $f: W \rightarrow W^{\prime}$ est un homéomorphisme entre deux voisinages $W$ et $W^{\prime}$ de 0 dans $\mathbf{R}^{2}$, préservant l'orientation et laissant fixe 0 , on dira que 0 est indifférent s'il vérifie la condition suivante :

Il existe un voisinage $V$ de 0 d'adhérence contenue dans $W$, tel que pour tout domaine de Jordan $U \subset V$ contenant 0 , la composante connexe $K$ de l'ensemble $\bigcap_{k \in \mathbf{Z}} f^{-k}(\bar{U})$ qui contient 0 rencontre $\partial U$.

Cette propriété ne dépend que de la classe de conjugaison du germe de $f$ en 0.

On peut caractériser les points indifférents par la condition équivalente :

Il existe un voisinage $V$ de 0 d'adhérence contenue dans $W$, tel que pour tout domaine de Jordan $U \subset V$ contenant 0 , l'ensemble $\bigcap_{k \in \mathbf{Z}} f^{-k}(\bar{U})$ rencontre $\partial U$.

Pour vérifier que ces deux conditions sont équivalentes, il suffit de remarquer que si $U$ ne vérifie pas la première condition, les ensembles $K$ et $\partial U$ appartiennent à deux composantes connexes distinctes de l'ensemble fermé

$$
X=\bigcap_{k \in \mathbf{Z}} f^{-k}(\bar{U}) \cup \partial U
$$

et peuvent donc être séparés par une courbe de Jordan disjointe de $X$ (voir [28], p. 143). Le domaine de Jordan $U^{\prime}$ bordé par cette courbe est alors contenu dans $U$ et il est isolant puisque

$$
\bigcap_{k \in \mathbf{Z}} f^{-k}\left(\bar{U}^{\prime}\right) \subset \bigcap_{k \in \mathbf{Z}} f^{-k}(\bar{U}) .
$$

\subsection{Points non indifférents}

D'après la remarque précédente, le point fixe 0 de $f: W \rightarrow W^{\prime}$ est non indifférent si et seulement s'il admet un système fondamental de voisinages qui sont des domaines de Jordan isolants. Comme cas particulier, on peut supposer que 0 admet un système fondamental de voisinages qui sont des domaines de Jordan dont la frontière est disjointe de leur image par $f$, c'est-à-dire de domaines attractifs ou répulsifs : on dira alors que 0 est dissipatif. Les cas les plus simples de points dissipatifs sont les puits et les sources. En dynamique complexe ce sont d'ailleurs les seuls cas possibles de points non indifférents. 


\subsection{Points-selles}

En dynamique réelle l'exemple le plus simple d'un point non indifférent non dissipatif est le cas d'une selle (éventuellement avec $p \geqslant 3$ demi-variétés stables alternant avec $p$ demi-variétés instables). Il est tentant d'appeler simplement point-selle tout point fixe qui est non indifférent et non dissipatif, c'est ce que nous ferons par la suite car la classification entre points fixes indifférents, point fixes dissipatifs et points-selles est la classification topologique qui nous intéressera dans cet article. Il faut cependant noter la remarque importante suivante :

Un point fixe elliptique générique d'un $C^{k}$-difféomorphisme préservant l'aire, $k$ assez grand, est un point-selle.

En effet, sous des hypothèses génériques, on peut appliquer la théorie KAM des courbes invariantes (voir M. Herman [19]), puis la théorie de Birkhoff sur les régions d'instabilité (voir [2]). On peut trouver dans chaque voisinage de 0 une région d'instabilité bordée par deux courbes invariantes $C^{-}$et $C^{+}$bordant des domaines de Jordan $U^{-}$et $U^{+}$vérifiant $0 \in U^{-} \subset \overline{U^{-}} \subset U^{+}$. Pour tout domaine de Jordan $U$ dont la frontière est contenue dans la région d'instabilité, c'està-dire vérifiant $\overline{U^{-}} \subset U \subset \bar{U} \subset U^{+}$, la composante connexe de $\bigcap_{k \in \mathbf{Z}} f^{-k}(\bar{U})$ qui contient 0 est égale à $\overline{U^{-}}$, c'est une conséquence de la théorie de Birkhoff.

Pour conclure, remarquons qu'en dynamique holomorphe, il n'y a pas de point-selle et qu'en dynamique hamiltonienne générique tous les points sont des points-selles.

\section{Calcul des $i\left(f^{k}, 0\right), k \geqslant 1$, dans le cas où 0 est accumulé ou non indifférent}

On se donne dans ce paragraphe un homéomorphisme $f: W \rightarrow W^{\prime}$ entre deux voisinages $W$ et $W^{\prime}$ de 0 préservant l'orientation et laissant fixe 0. On s'intéresse aux indices de Lefschetz $i\left(f^{k}, 0\right)$ des itérés de $f$, quand ils sont définis. On va rappeler ce qu'on sait sur ces indices.

\subsection{Le cas accumulé}

La démonstration du résultat suivant est due à S. Pelikan et E. Slaminka mais se trouve de façon un peu cachée dans [31].

Proposition 3.1. - Si 0 est accumulé et si c'est un point fixe isolé de $f^{k}$, alors $i\left(f^{k}, 0\right)=1$.

Démonstration. - Il suffit de montrer le résultat pour $k=1$. On peut trouver un homéomorphisme de $\mathbf{R}^{2}$ laissant fixe 0 et ayant même germe que $f$ en 0 que l'on note également $f$ pour ne pas alourdir les notations. L'ensemble

$$
X=\left\{z \in \mathbf{R}^{2} \mid f(z)=z \text { et } z \neq 0\right\}
$$

est fermé puisque 0 est isolé. On note $U$ la composante connexe de $\mathbf{R}^{2}-X$ contenant 0 et $\pi: \widetilde{U} \rightarrow U$ le revêtement universel de $U$. L'ensemble $\widetilde{U}$ est alors homéomorphe à $\mathbf{R}^{2}$. On choisit $z_{0} \in \pi^{-1}(\{0\})$ et on note $\tilde{f}$ le relèvement de $f_{\mid U}$ fixant $z_{0}$. Le germe de $\tilde{f}$ en chaque point fixe $z$ est conjugué au germe de $f$ en 0 et on a $i(\tilde{f}, z)=i(f, 0)$. L'indice de $\tilde{f}$ le long de toute courbe de Jordan sans point fixe $C$ est donc égal à $m i(f, 0)$, où $m$ est le nombre de points fixes de $\tilde{f}$ appartenant au domaine de Jordan bordé par $C$. Le point $z_{0}$ étant accumulé par hypothèse, l'homéomorphisme $\tilde{f}$ a une orbite périodique de période $q \geqslant 2$. D'après la proposition 1.1, on sait qu'il existe une courbe de $\operatorname{Jordan} C \subset \widetilde{U} \backslash \operatorname{Fix}(\tilde{f})$ telle que $i(\tilde{f}, C)=1$ : on en déduit que $i(f, 0)=1$. 


\subsection{Le cas dissipatif}

On sait également calculer les entiers $i\left(f^{k}, 0\right)$ dans le cas dissipatif.

Proposition 3.2. - Si 0 est dissipatif, alors $i\left(f^{k}, 0\right)=1$ si 0 est un point fixe isolé de $f^{k}$.

Démonstration. - La démonstration est très simple. On considère un domaine de Jordan contenant 0 , inclus dans $f^{-k}(W)$, ne contenant aucun autre point fixe de $f^{k}$ que 0 , et dont la frontière $C$ est disjointe de son image par $f$. Elle est également disjointe de son image par $f^{k}$ : l'indice de $f^{k}$ le long de $C$ est donc égal à 1 .

\subsection{Le cas des points-selles}

Le troisième cas, où l'on sait déterminer la suite $\left(i\left(f^{k}, 0\right)\right)_{k \in \mathbf{Z}}$ est le cas d'un point-selle non accumulé. Cette situation a été étudiée avec J.-C. Yoccoz [25] :

Proposition 3.3. - Si 0 est un point-selle non accumulé, il existe des entiers $q \geqslant 1$ et $r \geqslant 1$ tels que pour tout $k \geqslant 1$, on $a$ :

$$
\begin{cases}i\left(f^{k}, 0\right)=1 & \text { si } k \notin q \mathbf{Z}, \\ i\left(f^{k}, 0\right)=1-r q & \text { si } k \in q \mathbf{Z} .\end{cases}
$$

En conclusion, le cas où 0 est un point fixe indifférent non accumulé est le seul cas où la suite $\left(i\left(f^{k}, 0\right)\right)_{k \geqslant 1}$ (qui est bien définie) est encore indéterminée. C'est ce cas que nous étudierons principalement.

\section{Rappels sur les bouts premiers}

Dans ce paragraphe, et d'ailleurs dans tous ceux qui suivent, on se donne un homéomorphisme $f: W \rightarrow W^{\prime}$ entre deux voisinages $W$ et $W^{\prime}$ de 0 dans $\mathbf{R}^{2}$ préservant l'orientation et laissant fixe 0 . On supposera que $W$ et $W^{\prime}$ sont simplement connexes et on notera $\mathcal{D}$ l'ensemble des domaines de Jordan contenant 0 et dont l'adhérence est incluse dans $W$.

On note $\mathcal{K}$ l'ensemble des continus invariants par $f$, inclus dans $W$ et contenant 0 . On munit cet ensemble de la distance de Hausdorff. On définit également $\mathcal{K}^{*}=\mathcal{K} \backslash\{\{0\}\}$, qui est éventuellement vide.

\subsection{Compactification par bouts premiers}

Pour tout $K \in \mathcal{K}^{*}$ la composante connexe non bornée de $\mathbf{R}^{2}-K$, que l'on notera $U_{K}$, est annulaire, c'est-à-dire homéomorphe à $S^{1} \times \mathbf{R}$. Il existe une compactification naturelle du bout de $U_{K}$ correspondant à $K$, obtenue en ajoutant un ensemble homéomorphe à $S^{1}$, due à Carathéodory, appelée compactification par bouts premiers [7]. Cette compactification se définit de façon purement topologique, mais peut se comprendre par l'analyse complexe (voir [12] par exemple). On identifie $\mathbf{R}^{2}$ à $\mathbf{C}$ de la façon usuelle. On considère un difféomorphisme conforme

$$
h: U_{K} \rightarrow \mathbf{C} \backslash \overline{\mathbf{D}}
$$

(par exemple l'unique difféomorphisme tangent à l'identité à l'infini) et on munit $U_{K} \sqcup S^{1}$ de la topologie image réciproque de la topologie naturelle de $\mathbf{C} \backslash \mathbf{D}$ par l'application

$$
\bar{h}: U_{k} \sqcup S^{1} \rightarrow \mathbf{C} \backslash \mathbf{D}
$$


égale à $h$ sur $U_{k}$ et à l'identité sur $S^{1}$. L'espace topologique obtenu est homéomorphe à la compactification par bouts premiers de $U_{K}$, par abus de langage nous dirons que c'est la compactification par bouts premiers.

\subsection{Nombre de rotation}

Une propriété essentielle de la compactification par bouts premiers est le fait que la restriction de $f$ à $U_{K} \cap W$ se prolonge en un homéomorphisme défini au voisinage de $S^{1}$ (voir [27] par exemple). On note $\varphi_{K}$ l'homéomorphisme restreint au cercle, il préserve l'orientation et on peut définir son nombre de rotation $\rho_{K} \in \mathbf{T}^{1}=\mathbf{R} / \mathbf{Z}$.

\subsection{Nombre de rotation réel}

On notera

$$
\begin{aligned}
\Pi: \mathbf{R} \times] 0,+\infty[ & \rightarrow \mathbf{R}^{2}-\{0\}, \\
(\theta, r) & \mapsto(r \cos \theta, r \sin \theta)
\end{aligned}
$$

le revêtement universel de $\mathbf{R}^{2} \backslash\{0\}$ et

$$
T:(\theta, r) \mapsto(\theta+2 \pi, r)
$$

la transformation de recouvrement. On notera également

$$
\begin{aligned}
\Pi: \mathbf{R} & \rightarrow S^{1}, \\
\theta & \mapsto(\cos \theta, \sin \theta)
\end{aligned}
$$

le revêtement universel de $S^{1}$. Enfin, on définira

$$
\widetilde{W}=\Pi^{-1}(W), \quad \widetilde{W^{\prime}}=\Pi^{-1}\left(W^{\prime}\right),
$$

et

$$
\widetilde{U}_{K}=\Pi^{-1}\left(U_{K}\right)
$$

pour tout $K \in \mathcal{K}$.

L'application

$$
\Pi: \widetilde{U}_{K} \sqcup \mathbf{R} \mapsto U_{K} \sqcup S^{1},
$$

à valeurs dans le compactifié par bouts premiers de $U_{K}$, est le revêtement universel de celui-ci si on munit $\widetilde{U}_{K} \sqcup \mathbf{R}$ de la topologie image réciproque. À chaque relèvement $\tilde{f}: \widetilde{W} \rightarrow \widetilde{W}^{\prime}$ de $f_{\mid W-\{0\}}$ est associé naturellement un relèvement $\tilde{\varphi}_{K}$ de $\varphi_{K}$ à $\mathbf{R}$ et son nombre de rotation réel $\tilde{\rho}_{K}$, qui est un représentant réel de $\rho_{K}$.

\subsection{Arcs d'accès}

Rappelons d'abord quelques définitions.

Un arc d'accès de $K$ est un arc continu $\gamma:\left[0,1\left[\rightarrow U_{K}\right.\right.$ ayant une limite en 1 appartenant à $K$. On dit que le point limite est un point accessible de $K$. On sait alors - c'est une propriété de la compactification par bouts premiers - que l'arc $\gamma$ a également une limite dans $S^{1}$ (pour cette compactification). Deux arcs d'accès ayant même limite dans $S^{1}$ sont équivalents.

Les propriétés qui suivent sont classiques dans la théorie de Carathéodory. 
- Deux arcs d'accès $\gamma_{0}$ et $\gamma_{1}$ sont équivalents si et seulement s'ils ont même limite $z$ dans $K$ et s'il existe un arc d'accès $\gamma_{2}$ ayant également même limite dans $K$ et dont l'image rencontre l'image de $\gamma_{0}$ et de $\gamma_{1}$ dans tout voisinage de $z$.

- Tout arc d'accès est équivalent à un arc d'accès simple.

- L'ensemble des points accessibles est dense dans $\partial U_{K}$.

- L'ensemble des éléments de $S^{1}$, limites d'arcs d'accès de $K$, est dense dans $S^{1}$.

- L'ensemble des éléments de $S^{1}$, limites d'arcs d'accès de $K$ aboutissant en un point de $K$ différent de 0 , est également dense dans $S^{1}$.

\subsection{Arcs d'accès relevés}

Chaque arc d'accès de $K$ se relève en un arc à valeurs dans $\widetilde{U}_{K}=\pi^{-1}\left(U_{K}\right)$ et cet arc a une limite dans $\mathbf{R}$. Si $\tilde{\gamma}_{1}$ et $\tilde{\gamma}_{2}$ sont des relèvements d'arcs d'accès de $K$ et si $\tilde{x}_{1} \in \mathbf{R}$ et $\tilde{x}_{2} \in \mathbf{R}$ sont les limites respectives, on posera $\tilde{\gamma}_{1}<\tilde{\gamma}_{2}$ si $\tilde{x}_{1}<\tilde{x}_{2}$ et $\tilde{\gamma}_{1} \leqslant \tilde{\gamma}_{2}$ si $\tilde{x}_{1} \leqslant \tilde{x}_{2}$. Cette dernière relation définit un préordre sur l'ensemble des relèvements d'arcs d'accès et un ordre sur l'ensemble des "relèvements" des classes d'équivalence.

On peut définir de façon analogue un arc d'accès de $\widetilde{K}=\Pi^{-1}(K \backslash\{0\})$ à valeurs dans $\widetilde{U}_{K}$, c'est en particulier le relèvement d'un arc d'accès de $K$. La partie de $\mathbf{R}$ formée des points limites de tels arcs est également dense, on ne perd que les limites des relèvements d'arcs d'accès de $K$ aboutissant en 0 .

On utilisera les résultats suivants

LEMME 4.1. - Si $\left(\tilde{\gamma}_{i}\right)_{1 \leqslant i \leqslant n}$ est une famille de relèvements d'arc d'accès de K vérifiant

$$
\tilde{\gamma}_{1}<\tilde{\gamma}_{2}<\cdots<\tilde{\gamma}_{n}
$$

on peut trouver une famille $\left(a_{i}\right)_{1 \leqslant i \leqslant n}$ de réels vérifiant

$$
a_{1}<a_{2}<\cdots<a_{n}
$$

et une famille $\left(\widetilde{\Gamma}_{i}\right)_{1 \leqslant i \leqslant n}$ d'arcs définis sur $]-\infty, 1\left[\right.$ à valeurs dans $\widetilde{U}_{K}$, d'images disjointes deux à deux, tels que chaque $\widetilde{\Gamma}_{i}$ cö̈ncide avec $\tilde{\gamma}_{i}$ au voisinage de 1 et s'écrit $\widetilde{\Gamma}_{i}(t)=\left(a_{i},-t\right)$ pour $-t$ assez grand.

LEMME 4.2. - Si $\left(\tilde{\gamma}_{i}\right)_{1 \leqslant i \leqslant n}$ est une famille de relèvements d'arcs d'accès de $K$ à laquelle on peut associer une famille $\left(a_{i}\right)_{1 \leqslant i \leqslant n}$ de réels et une famille $\left(\widetilde{\Gamma}_{i}\right)_{1 \leqslant i \leqslant n}$ d'arcs vérifiant les propriétés ci-dessus, on a alors :

$$
\tilde{\gamma}_{1} \leqslant \tilde{\gamma}_{2} \leqslant \cdots \leqslant \tilde{\gamma}_{n}
$$

avec des inégalités strictes si les points limites sont tous distincts.

LEMME 4.3. - On ne peut pas trouver quatre arcs d'accès $\tilde{\gamma}_{1}, \tilde{\gamma}_{2}, \tilde{\gamma}_{3}, \tilde{\gamma}_{4}$ de $\widetilde{K}$ vérifiant

$$
\tilde{\gamma}_{1}<\tilde{\gamma}_{2}<\tilde{\gamma}_{3}<\tilde{\gamma}_{4}
$$

tels que $\tilde{\gamma}_{1}$ et $\tilde{\gamma}_{3}$ aient même limite $z \in \widetilde{K}$ et tels que $\tilde{\gamma}_{3}$ et $\tilde{\gamma}_{4}$ aient même limite $z^{\prime} \in \widetilde{K}$.

Démonstration. - Les deux premiers résultats se démontrent facilement. Limitons-nous à montrer la troisième. Considérons une famille $\left(a_{i}\right)_{1 \leqslant i \leqslant 4}$ de réels et une famille $\left(\widetilde{\Gamma}_{i}\right)_{1 \leqslant i \leqslant 4}$ d'arcs donnés par le premier lemme. La réunion $X$ de $\{z\}$ et des images de $\widetilde{\Gamma}_{1}$ et $\widetilde{\Gamma}_{3}$ sépare $\left.\mathbf{R} \times\right] 0,+\infty[$ en deux parties connexes, la réunion $X^{\prime}$ de $\left\{z^{\prime}\right\}$ et des images de $\widetilde{\Gamma}_{2}$ et $\widetilde{\Gamma}_{4}$ est connexe, disjointe de $X$ et rencontre chaque composante connexe de $\mathbf{R} \times] 0,+\infty[\backslash X$. 


\section{Dynamique et bouts premiers}

\subsection{Arcs positifs et négatifs}

On se fixe un relèvement $\tilde{f}: \widetilde{W} \rightarrow \widetilde{W}^{\prime}$ de $f_{\mid W \backslash\{0\}}$.

On dira qu'un arc d'accès $\gamma$ de $K$ à valeurs dans $W$ est un arc positif de $\tilde{f}$ si tout relèvement $\tilde{\gamma}$ de $\gamma$ vérifie $\tilde{f} \circ \tilde{\gamma} \geqslant \tilde{\gamma}$. On dira de même qu'un arc d'accès $\tilde{\gamma}$ de $\widetilde{K}$ à valeurs dans $\widetilde{W}$ est un arc positif de $\tilde{f}$ si $\tilde{f} \circ \tilde{\gamma} \geqslant \tilde{\gamma}$. De façon analogue, on parlera d'arc d'accès négatif, strictement positif et strictement négatif.

Le nombre de rotation $\tilde{\rho}_{K}$ est alors uniquement déterminé par les propriétés suivantes :

- Si $\frac{p}{q}<\tilde{\rho}_{K}$, alors tout arc d'accès de $K$ à valeurs dans $f^{-q+1}(W)$ est un arc strictement positif de $\tilde{f}^{q} \circ \widetilde{T}^{-p}$;

- Si $\frac{p}{q}>\tilde{\rho}_{K}$, alors tout arc d'accès de $K$ à valeurs dans $f^{-q+1}(W)$ est un arc strictement négatif de $\tilde{f}^{q} \circ \widetilde{T}^{-p}$.

\subsection{Un critère d'existence d'orbites périodiques}

Nous utiliserons le résultat suivant, classique dans la théorie des bouts premiers.

LEMME 5.1. - Si le nombre de rotation $\rho_{K}=p / q+\mathbf{Z}$ de $\varphi_{K}, K \in \mathcal{K}^{*}$, est rationnel et si $\varphi_{K}$ est semi-stable, alors la frontière de $U_{K}$ contient un point fixe de $f^{q}$.

Démonstration. - Choisissons un bout premier périodique de $K$. On sait qu'on peut trouver une suite $\left(\gamma_{n}\right)_{n \geqslant 0}$ de "cross-cuts" de ce bout premier dont le diamètre tend vers 0 (un "cross-cut" est un arc continu défini sur ]0, 1 [, à valeurs dans $U_{K}$ admettant une limite en 0 et en 1 dans $K$ et dont les limites dans le cercle des bouts premiers sont proches du bout premier considéré et entourent celui-ci. Comme la dynamique est semi-stable, on a $f^{q}\left(\gamma_{n}\right) \cap \gamma_{n} \neq \emptyset$ pour tout $n \geqslant 0$. Toute valeur d'adhérence de la suite $\left(\gamma_{n}\right)_{n \geqslant 0}$, pour la distance de Hausdorff est un point fixe de $f^{q}$ appartenant à $\partial\left(U_{K}\right)$.

Rappelons également le résultat suivant, conséquence immédiate de la densité dans $\partial U_{k}$ des points accessibles.

LEMme 5.2. - Si l'application $\varphi_{K}, K \in \mathcal{K}^{*}$, est périodique de période q, alors tout point de la frontière de $U_{K}$ est un point fixe de $f^{q}$.

\subsection{Ensembles $\mathcal{K}_{\rho}^{-}$et $\mathcal{K}_{\rho}^{+}$}

Pour tout nombre rationnel $\rho=\frac{p}{q}$, écrit sous forme irréductible, on note $\mathcal{K}_{\rho}^{-}\left(\right.$resp. $\left.\mathcal{K}_{\rho}^{+}\right)$ l'ensemble des continus $K \in \mathcal{K}^{*}$ tels que tout arc d'accès de $K$ à valeurs dans $f^{-q+1}(W)$ est un arc négatif (resp. positif) de $\tilde{f}^{q} \circ T^{-p}$. Un continu $K \in \mathcal{K}$ est donc dans $\mathcal{K}_{\rho}^{-}$(resp. $\mathcal{K}_{\rho}^{+}$) si et seulement si $\tilde{\varphi}_{K}^{q}(x) \leqslant x+p$ (resp. $\tilde{\varphi}_{K}^{q}(x) \geqslant x+p$ ) pour tout $x \in \mathbf{R}$, c'est-à-dire si et seulement si $\tilde{\rho}_{K}<\rho$ (resp. $\tilde{\rho}_{K}>\rho$ ) ou si $\tilde{\rho}_{K}=\rho$ et si la dynamique de $\varphi_{K}$ est semi-stable à gauche (resp. à droite).

Proposition 5.3. - Pour tout $\rho \in \mathbf{Q}$ les ensembles $\mathcal{K}_{\rho}^{-}$et $\mathcal{K}_{\rho}^{+}$sont des parties fermées de $\mathcal{K}^{*}$, stables par réunion finie.

Démonstration. - Il suffit d'établir le résultat quand $\rho=0$. Montrons d'abord que $\mathcal{K}^{*} \backslash \mathcal{K}_{0}^{-}$est ouvert dans $\mathcal{K}^{*}$. Si $K \notin \mathcal{K}_{0}^{-}$on peut trouver un arc d'accès $\gamma$ de $K$ strictement positif à valeurs dans $W$. Puisque l'ensemble des points limites dans $S^{1}$ des arcs d'accès n'aboutissant pas en 0 est dense dans $S^{1}$, on peut toujours supposer que $\gamma$ aboutit en un point $z \neq 0$. Choisissons alors 
un relèvement $\tilde{\gamma}$ de $\gamma$ et notons $\tilde{z} \in \widetilde{K}$ le point limite. D'après le lemme 4.1 , on peut trouver deux réels $a_{0}$ et $a_{1}$, avec $a_{0}<a_{1}$ et deux arcs d'accès de $\widetilde{K}$, notés $\widetilde{\Gamma}_{0}$ et $\widetilde{\Gamma}_{1}$, définis sur $]-\infty, 1[$, d'images disjointes, coïncidant respectivement avec $\tilde{\gamma}$ et $\tilde{f} \circ \tilde{\gamma}$ sur $] 1-\alpha, 1[$, où $\alpha \in] 0,1[$, et vérifiant

$$
\widetilde{\Gamma}_{0}(t)=\left(a_{0},-t\right) \quad \text { et } \quad \widetilde{\Gamma}_{1}(t)=\left(a_{1},-t\right),
$$

si $-t$ est grand.

Munissons $\mathbf{R} \times] 0,+\infty[$ de la distance euclidienne usuelle et considérons $\varepsilon>0$ tel que :

- $B(\tilde{z}, \varepsilon) \subset \widetilde{W}$,

- $B(\tilde{f}(\tilde{z}), \varepsilon) \subset \mathbf{R} \times] 0,+\infty[$,

- $\left.\left.\widetilde{\Gamma}_{0}(]-\infty, 1-\alpha\right]\right) \cap B(\tilde{z}, \varepsilon)=\emptyset$,

- $\left.\left.\widetilde{\Gamma}_{1}(]-\infty, 1-\alpha\right]\right) \cap B(\tilde{f}(\tilde{z}), \varepsilon)=\emptyset$,

- $\left(\widetilde{\Gamma}_{0}(]-\infty, 1[) \cup B(\tilde{z}, \varepsilon)\right) \cap\left(\widetilde{\Gamma}_{1}(]-\infty, 1[) \cup B(\tilde{f}(\tilde{z}), \varepsilon)\right)=\emptyset$.

Choisissons ensuite $\left.\varepsilon^{\prime} \in\right] 0, \varepsilon[$ tel que :

$$
\tilde{f}\left(B\left(\tilde{z}, \varepsilon^{\prime}\right)\right) \subset B(\tilde{f}(\tilde{z}), \varepsilon)
$$

et notons $\widetilde{\Gamma}_{0}\left(t^{\prime}\right)$ le premier point où $\widetilde{\Gamma}_{0}$ atteint $\overline{B\left(\tilde{z}, \varepsilon^{\prime}\right)}$.

Si $K^{\prime} \in \mathcal{K}^{*}$ est assez proche de $K$, alors $\widetilde{K}^{\prime}$ est disjoint de $\left.\left.\widetilde{\Gamma}_{0}(]-\infty, t^{\prime}\right]\right)$ et de $\left.\left.\widetilde{\Gamma}_{1}(]-\infty, t^{\prime}\right]\right)$ et on a $\widetilde{K}^{\prime} \cap B\left(\tilde{z}, \varepsilon^{\prime}\right) \neq \emptyset$. Choisissons un point dans $\widetilde{K}^{\prime} \cap B\left(\tilde{z}, \varepsilon^{\prime}\right)$, considérons le segment joignant $\widetilde{\Gamma}_{0}\left(t^{\prime}\right)=\gamma\left(t^{\prime}\right)$ à ce point. Ce segment définit naturellement un arc affine $\tilde{\gamma}^{\prime}$ défini sur $\left[t^{\prime}, 1[\right.$, à valeurs dans $\widetilde{U}_{K^{\prime}}$ et aboutissant au premier point de $\widetilde{K}^{\prime}$ rencontré par le segment. Prolongeons cet arc par $\widetilde{\Gamma}_{0}$ sur $\left.]-\infty, t^{\prime}\right]$. Prolongeons de même l'arc $\tilde{f} \circ \tilde{\gamma}^{\prime}$ par $\widetilde{\Gamma}_{1}$ sur $\left.]-\infty, t^{\prime}\right]$. Les deux arcs maintenant définis sur $]-\infty, 1[$ sont d'images disjointes et leurs points limites distincts. D'après le lemme 4.2 , on en déduit que $\tilde{\gamma}^{\prime}$ est un arc d'accès strictement positif de $\widetilde{K}^{\prime}$, ainsi $K^{\prime} \notin \mathcal{K}_{0}^{-}$.

La seconde partie de la proposition est presque évidente. Soient $K_{1}$ et $K_{2}$ deux éléments de $\mathcal{K}^{*}$ dont la réunion $K$ n'appartient pas à $\mathcal{K}_{0}^{-}$. On construit comme précédemment des arcs $\tilde{\gamma}, \widetilde{\Gamma}_{0}$ et $\widetilde{\Gamma}_{1}$. Le point $z$ appartient à $K_{1}$ ou à $K_{2}$. S'il appartient à $K_{1}$ (resp. à $K_{2}$ ), l'arc $\tilde{\gamma}$ est alors un arc d'accès de $K_{1}$ (resp. $K_{2}$ ) strictement positif.

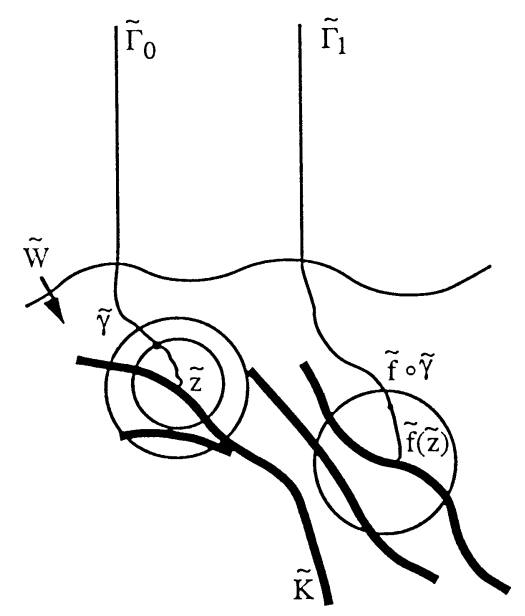

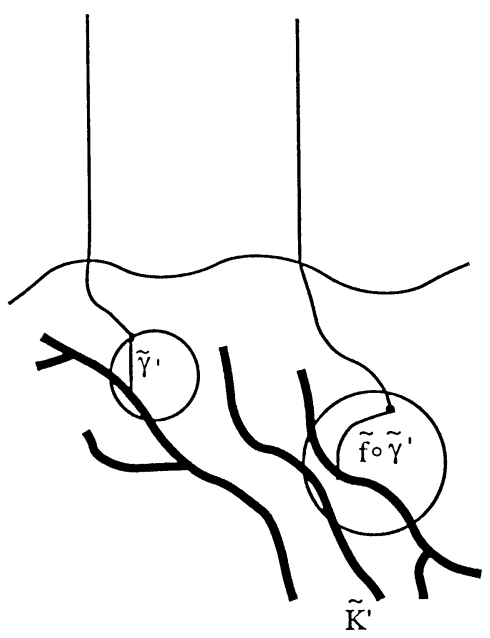

Fig. 1. 

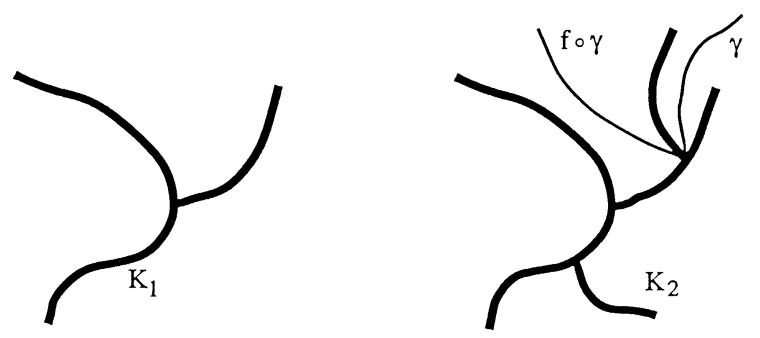

Fig. 2.

Remarque. - Du dernier raisonnement, on déduit que si $K_{1}$ est inclus dans $K_{2}$, alors tout arc d'accès strictement positif de $K_{2}$ aboutissant en un point de $K_{1}$ est un arc d'accès positif de $K_{1}$ (mais non nécessairement strictement positif). En particulier si $K_{1} \subset K_{2}$ et si $K_{1}$ et $K_{2}$ ont un arc d'accès en commun, alors $\tilde{\rho}_{K_{1}}=\tilde{\rho}_{K_{2}}$.

\subsection{Applications}

On déduit immédiatement de la proposition 5.3 :

Corollaire 5.4. - L'application $K \rightarrow \tilde{\rho}_{K}$ est continue sur $\mathcal{K}^{*}$.

On en déduit également :

Corollaire 5.5. - Si $\mathcal{K}^{\prime}$ est une partie compacte de $\mathcal{K}$ stable par réunion finie et si $\left(K_{\alpha}\right)_{\alpha \in A}$ est une famille d'éléments de $\mathcal{K}_{\rho}^{-} \cap \mathcal{K}^{\prime}$, alors $\overline{\bigcup_{\alpha \in A} K_{\alpha}} \in \mathcal{K}_{\rho}^{-} \cap \mathcal{K}^{\prime}$.

Démonstration. - L'ensemble $\overline{\bigcup_{\alpha \in A} K_{\alpha}}$ est limite, suivant le filtre des parties finies $I$ de $A$, des ensembles $\bigcup_{\alpha \in I} K_{\alpha}$, qui appartiennent tous à $\mathcal{K}_{\rho}^{-} \cap \mathcal{K}^{\prime}$.

Par le lemme de Zorn, on en déduit alors :

Corollaire 5.6. - Toute partie compacte de $\mathcal{K}$ stable par réunion finie, qui contient un élément de $\mathcal{K}_{\rho}^{-}$(resp. de $\mathcal{K}_{\rho}^{+}$), contient un élément de $\mathcal{K}_{\rho}^{-}$(resp. de $\mathcal{K}_{\rho}^{+}$) maximal pour l'inclusion.

\section{Une propriété des points indifférents non accumulés}

On supposera dans ce paragraphe que 0 est un point fixe indifférent non accumulé. On fixe alors un domaine de Jordan $U \in \mathcal{D}$ ne contenant aucune orbite périodique autre que $\{0\}$, et tel que pour tout domaine de Jordan $U^{\prime}$ inclus dans $U$ et contenant 0 , la composante connexe de $\bigcap_{k \in \mathbf{Z}} f^{-k}\left(\bar{U}^{\prime}\right)$ qui contient 0 rencontre $\partial U^{\prime}$. On note $K_{0}$ la composante connexe de $\bigcap_{k \in \mathbf{Z}} f^{-k}(\bar{U})$ qui contient 0 . On fixe ensuite un relèvement $\tilde{f}: \widetilde{W} \rightarrow \widetilde{W}^{\prime}$ de $f_{\mid W \backslash\{0\}}$. On va montrer le résultat suivant :

\section{Proposition 6.1. - Si K est un élément de $\mathcal{K}^{*}$ inclus dans $\bar{U}$, alors $\tilde{\rho}_{K}=\tilde{\rho}_{K_{0}}$.}

Si $\left(\varphi_{t}\right)_{t \in[0,1]}$ est une famille d'homéomorphismes de $S^{1}$ dépendant continûment de $t$ et si la famille des nombres de rotation n'est pas constante, on peut trouver des valeurs de $t$ pour lesquelles $\varphi_{t}$ est semi-stable à gauche. On aura ici une situation analogue. On utilisera dans la démonstration 
- la continuité de la fonction $K \mapsto \rho_{K}$;

- un argument de "connexité";

- le lemme 5.1 qui étudie le cas où $\varphi_{K}$ est semi-stable.

Lemme 6.1. - Si $K \in \mathcal{K}^{*}$ est un continu plein inclus dans $U$, il existe alors une suite $\left(K_{n}\right)_{n \geqslant 0}$ strictement décroissante d'éléments de $\mathcal{K}^{*}$ tels que

$$
K=\bigcap_{n \geqslant 0} K_{n}=\lim _{n \rightarrow+\infty} K_{n}
$$

Démonstration. - On peut écrire $K=\bigcap_{n \geqslant 0} \bar{U}_{n}$ où $U_{n}$ est une suite de domaines de Jordan vérifiant $U_{0}=U$ et $\bar{U}_{n+1} \subset U_{n}$ pour tout $n \geqslant 0$. On en déduit que $K=\bigcap_{n \geqslant 0} K_{n}$ où $K_{n}$ est la composante connexe de 0 dans $\bigcap_{k \in \mathbf{Z}} f^{-k}\left(\bar{U}_{n}\right)$. Cette suite est strictement décroissante puisque $K_{n} \cap \partial U_{n} \neq \emptyset$ pour tout $n \geqslant 0$.

Démonstration de la proposition 6.1. - Supposons que $K \in \mathcal{K}^{*}$ soit inclus dans $\bar{U}$ (et donc dans $K_{0}$ ) et que l'on ait $\tilde{\rho}_{K}<\tilde{\rho}_{K_{0}}$. Choisissons alors deux nombres rationnels $\tilde{\rho}_{1}$ et $\tilde{\rho}_{2}$ vérifiant

$$
\tilde{\rho}_{K}<\tilde{\rho}_{1}<\tilde{\rho}_{2}<\tilde{\rho}_{K_{0}}
$$

et tels que $q_{1}$ et $q_{2}$ soient premiers entre eux, si on écrit $\tilde{\rho}_{1}=p_{1} / q_{1}$ et $\tilde{\rho}_{2}=p_{2} / q_{2}$ sous forme irréductible.

L'ensemble

$$
\mathcal{K}^{\prime}=\left\{K^{\prime} \in \mathcal{K} \mid K \subset K^{\prime} \subset K_{0}\right\}
$$

est compact, stable par réunion finie et intersecte $\mathcal{K}_{\rho_{1}}^{-}$puisqu'il contient $K$ : il contient donc un élément maximal $K_{1}$, d'après le corollaire 5.6. Celui-ci est plein puisqu'il est maximal et que $K_{0}$ est plein, de plus il est contenu dans $U$ d'après la remarque qui suit la proposition 5.3. De la continuité de l'application $K \mapsto \tilde{\rho}_{K}$ et du lemme 6.1 , on déduit que l'on a $\tilde{\rho}_{K_{1}}=\tilde{\rho}_{1}$ et que la dynamique de $\varphi_{K_{1}}$ est semi-stable à gauche. De façon analogue on peut trouver $K_{2} \in \mathcal{K}^{*}$, compact plein vérifiant

$$
K_{1} \subset K_{2} \subset K_{0}
$$

avec $\tilde{\rho}_{K_{2}}=\tilde{\rho}_{2}$ et tel que la dynamique de $\tilde{\varphi}_{K_{2}}$ soit semi-stable à gauche. Remarquons que $K_{1}$ et $K_{2}$ n'ont pas d'arcs d'accès en commun puisque $K_{2} \subset K_{1}$ et que $\tilde{\rho}_{2} \neq \tilde{\rho}_{1}$.

Identifions là encore $\mathbf{R}^{2}$ et $\mathbf{C}$ et notons $h: U_{K_{1}} \rightarrow \mathbf{R}^{2} \backslash \overline{\mathbf{D}}$ le difféomorphisme conforme tangent à l'identité en l'infini. L'homéomorphisme $h \circ f \circ h^{-1}$, défini sur $h\left(W \backslash K_{1}\right)$ se prolonge sur $S^{1}$ en $\varphi_{K_{1}}$, on le prolonge de façon arbitraire sur $\mathbf{D}$ : on a alors un homéomorphisme $\hat{f}$ entre $h\left(W \backslash K_{1}\right) \cup \overline{\mathbf{D}}$ et $h\left(W^{\prime} \backslash K_{1}\right) \cup \overline{\mathbf{D}}$ conjugué à $f_{\mid W \backslash K_{1}}$ en dehors de $\overline{\mathbf{D}}$.

On sait, d'après le lemme 1.2, que l'adhérence de toute composante connexe de $K_{2} \backslash K_{1}$ rencontre $K_{1}$. On en déduit que l'ensemble

$$
\widehat{K}_{2}=h_{1}\left(K_{2} \backslash K_{1}\right) \cup \overline{\mathbf{D}}
$$

est un continu invariant par $\hat{f}$.

LEMME 6.2.- L'ensemble des points limites, dans le cercle des bouts premiers de $\widehat{K}_{2}$, des arcs d'accès de $\widehat{K}_{2}$ aboutissant en un point de $\widehat{K}_{2} \backslash \overline{\mathbf{D}}$, est dense. 
Démonstration. - Remarquons d'abord que tout point de $S^{1} \subset \widehat{K}_{2}$ est adhérent à $\widehat{K}_{2} \backslash \overline{\mathbf{D}}$. En effet dans le cas contraire on peut trouver une boule ouverte $V$ disjointe de $\widehat{K}_{2} \backslash \overline{\mathbf{D}}$ et intersectant $S^{1}$ en un intervalle ouvert non trivial $I$. Puisque l'ensemble des points limites, dans le cercle des bouts premiers de $K_{1}$, des arcs d'accès de $K_{1}$ est dense, il existe un arc d'accès de $K_{1}$ dont l'image par $h_{1}$ est contenue dans $U$ et aboutit en un point de $I$. Cet arc est alors un arc d'accès commun à $K_{1}$ et $K_{2}$, ce qui est impossible.

Donnons-nous maintenant un intervalle ouvert non trivial $I$ du cercle des bouts premiers de $\widehat{K}_{2}$ et choisissons dans cet arc deux points distincts, chacun point limite d'un arc d'accès de $\widehat{K}_{2}$. Nous allons montrer que dans l'intervalle fermé $J \subset I$ délimité par ces deux points, il existe un point limite d'un arc d'accès de $\widehat{K}_{2}$ aboutissant en un point de $\widehat{K}_{2} \backslash \overline{\mathbf{D}}$. Si aucune des deux extrémités n'a cette propriété, on peut trouver un arc simple $\gamma:] 0,1\left[\rightarrow \mathbf{R}^{2}\right.$, à valeurs dans $\mathbf{R}^{2} \backslash \widehat{K}_{2}$ ayant une limite en 0 et en 1 (dans $\mathbf{R}^{2}$ ) appartenant à $S^{1}$ et ayant les deux extrémités de $J$ comme limites dans le cercle des bouts premiers de $\widehat{K}_{2}$. L'arc $\gamma$ sépare $\mathbf{R}^{2} \backslash \bar{U}_{\widehat{K}_{2}}$ en deux composantes connexes et nous venons de montrer que chacune d'entre elles rencontre $\widehat{K}_{2} \backslash \overline{\mathbf{D}}$. On peut choisir dans chacune de ces composantes un arc d'accès de $\widehat{K}_{2}$ aboutissant en un point de $\widehat{K}_{2} \backslash \overline{\mathbf{D}}$ : le point limite, dans le cercle des bouts premiers de $\widehat{K}_{2}$, de l'un de ces deux arcs doit être dans $I$.

Fin de la démonstration de la proposition 6.1. - Si $\gamma$ est un arc d'accès de $K_{2}$, à valeurs dans $W \backslash K_{2}$, son image par $h$ est un arc d'accès de $\widehat{K}_{2}$, puisque le point limite n'appartient pas à $K_{1}$. L'ensemble des arcs images obtenus de cette façon est formée des arcs d'accès de $\widehat{K}_{2}$ à valeurs dans $h\left(W \backslash K_{2}\right)$ et aboutissant en un point de $\widehat{K}_{2} \backslash \bar{D}$.

Cette application entre arcs d'accès de $K_{2}$ à valeurs dans $W \backslash K_{2}$ et arcs d'accès de $\widehat{K}_{2}$ est compatible avec les relations d'équivalences sur les arcs d'accès et définit une application naturelle entre les classes d'équivalence d'arcs d'accès de $K_{2}$ et les classes d'équivalences d'arcs d'accès de $\widehat{K}_{2}$. Pour s'en convaincre, il suffit d'utiliser la propriété caractéristique donnée au paragraphe 4 de deux arcs équivalents. Cette propriété nous dit également que l'application induite sur les classes d'équivalence est injective et que son image est formée des classes d'équivalence d'arcs aboutissant en un point de $\widehat{K}_{2} \backslash \bar{D}$. Cette application induit donc une injection naturelle $H$ entre une partie dense du cercle des bouts premiers en $K_{2}$ et une partie du cercle des bouts premiers en $\widehat{K}_{2}$, qui est également dense d'après le lemme 6.2.

Utilisant la caractérisation liée à l'ordre donnée au paragraphe 4, il est facile de voir que cette application se relève naturellement aux revêtements universels des cercles et que ce relèvement est une application strictement croissante.

Si on note respectivement $\varphi_{K_{2}}$ et $\hat{\varphi}_{\widehat{K}_{2}}$ les applications définies naturellement par $f$ et $\hat{f}$ sur les cercles de bouts premiers. On a alors

$$
\hat{\varphi}_{\widehat{K}_{2}} \circ H=H \circ \varphi_{K_{2}} .
$$

On déduit de tout ceci que $\hat{\varphi}_{\widehat{K}_{2}}$ a même nombre de rotation que $\varphi_{K_{2}}$ et comme $H$ est d'image dense, on en déduit que la dynamique de $\hat{\varphi}_{\widehat{K}_{2}}$ est semi-stable à gauche.

On utilise maintenant le lemme 5.1. On sait que $\hat{f}^{q_{2}}$ a un point fixe sur $\partial U_{\widehat{K}_{2}}$. Comme $q_{1}$ et $q_{2}$ sont premiers entre eux, ce point appartient à $h\left(K_{2} \backslash K_{1}\right)$ et on en déduit que $K_{2} \backslash K_{1}$ contient un point périodique et donc une orbite périodique. Ceci contredit les hypothèses.

Une démonstration en tout point analogue donne le résultat suivant :

Proposition 6.2. - Si 0 est un point indifférent non dégénéré, alors l'application $K \mapsto \tilde{\rho}_{K}$ admet une limite quand $K$ tend vers $\{0\}$. 
On déduit de ce paragraphe que l'on peut définir le nombre de rotation, élément de $\mathbf{T}^{1}$, d'un point fixe indifférent non dégénéré, c'est la limite de $\rho_{K}$ quand $K$ tend vers $\{0\}$. Ce nombre est égal à la valeur commune des $\rho_{K}, K$ proche de $\{0\}$, dans le cas où 0 est non accumulé.

\section{7. Éclatements}

\section{1. Éclatements, symétrisé d'un éclatement}

On appelle éclatement de $K \in \mathcal{K}$, ou $K$-éclatement toute compactification du bout de l'anneau $U_{K}$ correspondant à $K$, obtenue en ajoutant le cercle $S^{1}$, telle que $f$ se prolonge en un homéomorphisme au voisinage de $S^{1}$. La restriction $\varphi$ de $f$ à $S^{1}$ préserve alors l'orientation et admet un nombre de rotation.

Tout homéomorphisme $\varphi^{\prime}$ de $S^{1}$ vérifiant $\varphi^{\prime} \circ h=h \circ \varphi$, où $h: S^{1} \rightarrow S^{1}$ est une application continue de degré 1 , définit naturellement un nouvel éclatement dont $\varphi^{\prime}$ est l'application au bord. On dira que cet éclatement est un facteur de l'éclatement initial (par abus de langage, on dira que l'éclatement $\varphi^{\prime}$ est un facteur de l'éclatement $\varphi$ ). En particulier, $\varphi^{\prime}$ à même nombre de rotation que $\varphi$.

On a rencontré les éclatements par bouts premiers, on peut aussi dans le cas où $f$ est un difféomorphisme, éclater le point fixe en ajoutant le cercle des demi-droites issues de ce point. On peut également construire des facteurs de tels éclatements, par exemple si l'homéomorphisme au bord a un nombre de rotation rationnel ou alors s'il a un nombre de rotation irrationnel et s'il n'est pas minimal.

Étant donné un éclatement, on peut construire un espace topologique $A$ en prenant deux copies du compactifié que l'on recolle par l'identité sur le cercle $S^{1}$. On obtient un ensemble homéomorphe à un anneau ouvert et un homéomorphisme $F$, le symétrisé de l'éclatement, défini au voisinage d'une courbe fermée invariante non homotope à zéro. On notera $\sigma$ l'involution naturellement définie sur $A$.

\section{2. Éclatements non indifférents}

On dira qu'un éclatement est fini si l'application au bord $\varphi$ a un nombre fini d'orbites périodiques.

On dira qu'un éclatement $\varphi$ est indifférent s'il n'est pas fini ou alors s'il est fini et s'il existe un point périodique de $\varphi$ qui est un point fixe indifférent de $F^{q}$, où $q$ est la période commune de ces orbites périodiques. Dans le cas contraire on dira qu'il est non indifférent. Un éclatement est donc non indifférent s'il est fini et si chaque point périodique de $\varphi$ est un point fixe non indifférent de $F^{q}$.

S'il est clair que tout facteur d'un éclatement fini est encore fini, nous avons le résultat suivant :

\section{LEMME 7.1. - Tout facteur $\varphi^{\prime}$ d'un K-éclatement fini indifférent $\varphi$ est indifférent.}

Démonstration. - Supposons que $\varphi^{\prime}$ ne soit pas indifférent et montrons qu'il en est de même de $\varphi$. La semi-conjugaison $h: S^{1} \rightarrow S^{1}$ entre $\varphi$ et $\varphi^{\prime}$ se prolonge en une semi-conjugaison de même nom $h: A \rightarrow A^{\prime}$ entre les symétrisés des compactifiés, égale à l'identité sur les deux copies de $U_{K}$. Soit $z$ un point fixe de $\varphi$, qui est isolé puisque $\varphi$ est fini, et $z^{\prime}$ l'image de $z$ par $h$. On peut considérer un système (topologique) de coordonnées polaires $(\theta, r) \in \mathbf{T}^{1} \times[-1,+1]$ au voisinage du cercle $S^{1} \in A$ vérifiant $\sigma(\theta, r)=(\theta,-r)$. Notons $\left(\theta_{0}, 0\right)$ les coordonnées de $z$ et fixons $\varepsilon_{0}>0$ petit. 
Pour tout $\varepsilon \in] 0, \varepsilon_{0}\left[\right.$, il existe $\varepsilon^{\prime}=\nu(\varepsilon)>0$ tel que l'image ou l'image inverse par $F$ de chacun des segments

$$
\left\{\theta_{0}-\varepsilon\right\} \times[-\varepsilon, \varepsilon]
$$

et

$$
\left\{\theta_{0}+\varepsilon\right\} \times[-\varepsilon, \varepsilon]
$$

est disjointe du rectangle

$$
\left[\theta_{0}+\varepsilon, \theta_{0}+\varepsilon\right] \times\left[-\varepsilon^{\prime}, \varepsilon^{\prime}\right]
$$

Soit $\left.\varepsilon \in] 0, \varepsilon_{0}\right]$. Puisque $z^{\prime}$ n'est pas indifférent, on peut trouver un domaine de Jordan $\Delta^{\prime}$, voisinage isolant de $z^{\prime}$ tel que $\left.h^{-1}\left(\Delta^{\prime}\right) \subset \mathbf{T}^{1} \times\right]-\nu(\varepsilon),+\nu(\varepsilon)$ [. Puisque les points du cercle $S^{1} \subset A^{\prime}$, dont la préimage est un intervalle non trivial de $S^{1} \subset A$, est dénombrable, on peut supposer, quitte à perturber la frontière de $\Delta^{\prime}$, que celle-ci rencontre le cercle $S^{1} \subset A^{\prime}$ en un nombre fini de points et que chacun d'eux n'a qu'un antécédent par $h$. L'ensemble $h^{-1}(\Delta)$ est alors un domaine de Jordan et il est isolant. Un résultat de Kérékjártó [22] (voir également [24]) nous dit que toute composante connexe de l'intersection de deux domaines de Jordan est un domaine de Jordan, en particulier la composante connexe $\Delta$ de

$$
\left.h^{-1}\left(\Delta^{\prime}\right) \cap\right] \theta_{0}-\varepsilon, \theta_{0}+\varepsilon[\times]-\varepsilon, \varepsilon[
$$

qui contient $z$. La frontière de $\Delta$ est formée de points de la frontière de $h^{-1}\left(\Delta^{\prime}\right)$ et de points des segments

$$
\left\{\theta_{0}-\varepsilon\right\} \times[-\varepsilon, \varepsilon]
$$

et

$$
\left\{\theta_{0}+\varepsilon\right\} \times[-\varepsilon, \varepsilon]
$$

Puisque $h^{-1}\left(\Delta^{\prime}\right)$ est isolant, aucun point de sa frontière n'a son orbite entièrement contenue dans $\bar{\Delta}$. Comme il en est de même de tout point appartenant aux segments

$$
\left\{\theta_{0}-\varepsilon\right\} \times[-\varepsilon, \varepsilon]
$$

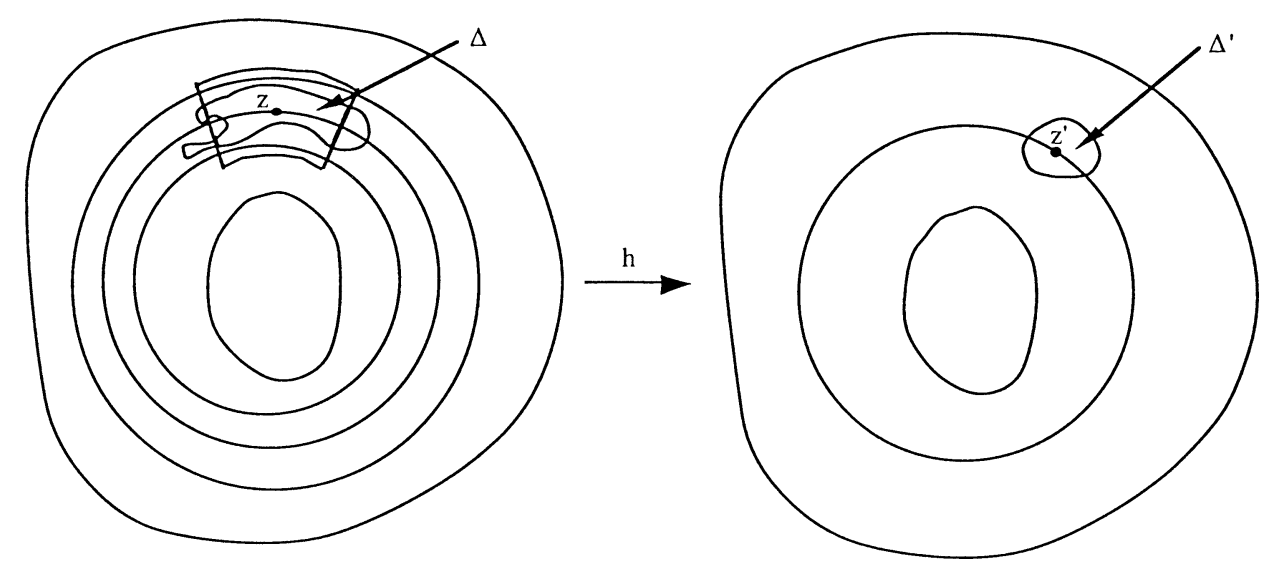

Fig. 3. 
et

$$
\left\{\theta_{0}+\varepsilon\right\} \times[-\varepsilon, \varepsilon]
$$

le domaine de Jordan $\Delta$ est isolant. On a montré que $z$ est non indifférent.

\subsection{Construction d'éclatements non indifférents}

Il est facile de construire un éclatement fini à partir d'un éclatement $\varphi$ qui n'est pas périodique. Si l'éclatement $\varphi$ n'est pas fini et a un nombre de rotation $p / q$, avec $p$ et $q$ premiers entre eux, on considère un ensemble invariant par $\varphi$ de $n q$ composantes connexes distinctes $I_{k}, 1 \leqslant k \leqslant n q$, du complémentaire de l'ensemble des points périodiques. On a une semi-conjugaison naturelle envoyant chaque composante connexe de $S^{1}-\bigcup_{1 \leqslant k \leqslant n q} I_{k}$ sur un point périodique. Le résultat qui suit indique comment construire un éclatement non indifférent.

Proposition 7.2.- Soit $U \in \mathcal{D}$. On suppose que la composante connexe $K$ de $\bigcap_{k \in \mathbf{Z}} f^{-k}(\bar{U})$ contenant 0 rencontre $\partial U$ et que $\partial U$ ne contient aucun point périodique dont l'orbite est contenue dans $\bar{U}$. Alors l'éclatement $\varphi_{K}$ défini par les bouts premiers a un facteur non indifférent.

Démonstration. - Si le nombre de rotation $\rho_{K}$ de $\varphi_{K}$ n'appartient pas à $\mathbf{Q} / \mathbf{Z}$, il n'y a pas de point périodique et $\varphi_{K}$ est non indifférent. On supposera donc que $\rho_{K}=0$, le cas rationnel se traitant de même. Grâce au théorème de Schoënflies, on peut toujours supposer que $\partial U$ est le cercle $S^{1}$.

Pour tout $z \in K \cap \partial U$, le rayon externe

$$
\begin{aligned}
\gamma_{z}:[0,1[ & \rightarrow \mathbf{R}^{2}, \\
t & \mapsto(2-t) z
\end{aligned}
$$

est un arc d'accès de $K$ aboutissant en $z$. Tout arc d'accès de $K$ aboutissant en $z$ et à valeurs dans $\mathbf{R}^{2} \backslash U$ est clairement équivalent à $\gamma_{z}$. Notons $l(z) \in S^{1}$ le point limite de $\gamma_{z}$ pour la compactification en bouts premiers. La fonction $l$ définie sur $K \cap \partial U$ est alors continue et injective et induit un homéomorphisme entre la partie compacte $K \cap \partial U$ et une partie fermée de $S^{1}$ disjointe de l'ensemble $\operatorname{Fix}\left(\varphi_{K}\right)$ des points fixes de $\varphi_{K}$, d'après les hypothèses faites sur $\partial U$. La continuité de $l$ est une propriété classique des bouts premiers, on peut la montrer par exemple en passant au revêtement universel, en utilisant la relation d'ordre et les lemmes 4.1 et 4.2. De la même façon, on montre que la réunion de $\partial U \backslash K$ et de $l(K \cap \partial U)$ est une courbe fermée simple non homotope à zéro du compactifié par bouts premiers de $U_{K}$.

D'après les hypothèses, $l(K \cap \partial U)$ et $\operatorname{Fix}\left(\varphi_{K}\right)$ sont deux parties fermées disjointes, il existe donc une famille finie $\left(I_{k}\right)_{1 \leqslant k \leqslant n}$ de composantes connexes disjointes de $S^{1} \backslash$ Fix $\left(\varphi_{K}\right)$ dont la réunion contient l'image de $l$. Nous considérons le facteur $\varphi$ de $\varphi_{K}$ défini naturellement par cette famille, nous allons montrer que l'éclatement fini $\varphi$ est non indifférent.

Remarquons que la réunion de $\partial U \backslash K$ et de $l(K \cap \partial U)$ est encore une courbe fermée simple $C$ dans le nouveau compactifié. Notons $A$ l'anneau défini par recollement de deux copies du compactifié, $\sigma$ l'involution naturelle définie sur $A$ et $F$ le symétrisé. Soit $z^{\prime} \in S^{1}$ un point fixe de $\varphi$ et $V$ un voisinage de $z$ disjoint de $C \cup \sigma(C)$, invariant par $\sigma$, assez petit pour que l'orbite de tout point de $V \cap S^{1}$ autre que $z^{\prime}$, sorte de $V$. Si $K^{\prime}$ est une partie compacte connexe de $A$ invariante par $F$, contenant $z^{\prime}$, ne se réduisant pas à ce point, et contenue dans $V$, il en est de même de $K^{\prime} \cup \sigma\left(K^{\prime}\right)$. Comme l'adhérence de toute composante connexe de $K^{\prime} \cup \sigma\left(K^{\prime}\right) \backslash\left\{z^{\prime}\right\}$ contient $z^{\prime}$ (c'est le lemme 1.2) et comme $K^{\prime} \cup \sigma\left(K^{\prime}\right)$ ne rencontre $S^{1}$ qu'au point $z^{\prime}$, on peut écrire

$$
K^{\prime} \cup \sigma\left(K^{\prime}\right)=K^{\prime \prime} \cup \sigma\left(K^{\prime \prime}\right)
$$



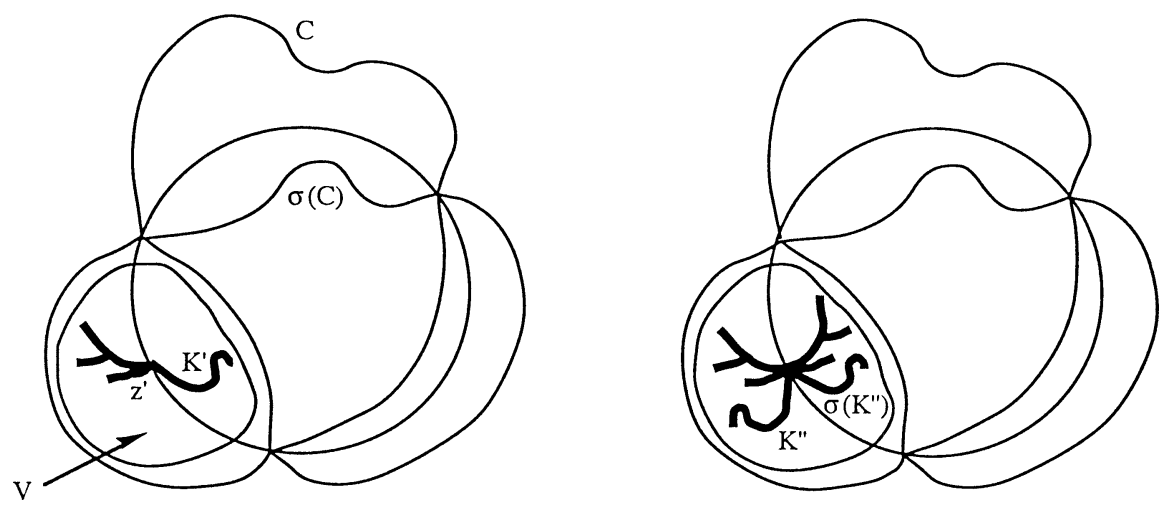

Fig. 4.

où $K^{\prime \prime}=K^{\prime} \cap\left(U_{K} \sqcup S^{1}\right)$ est un continu contenu dans le compactifié par bouts premiers original $U_{K} \sqcup S^{1}$ de $U_{K}$ et ne rencontre $S^{1}$ qu'en $z^{\prime}$. Toujours pour les mêmes raisons, l'ensemble $K^{\prime \prime} \backslash\{z\} \cup K$ est une partie compacte connexe invariante par $f$. Comme elle est contenue dans $\bar{U}$ et comme elle est strictement plus grande que $K$, on a une contradiction.

Remarquons en particulier que si $\varphi_{K}$ est fini, il est non indifférent.

\section{8. Éclatements et indices}

\subsection{Calcul d'indice}

Commençons par un résultat classique :

Proposition 8.1. - Soit $\varphi$ un éclatement fini de $K \in \mathcal{K}$. Si $U \in \mathcal{D}$ contient $K$ et si $\bar{U} \backslash K$ n'a pas de point fixe, alors on a:

$$
i(f, \partial U)=1+\frac{1}{2} \sum_{z \in \operatorname{Fix} \varphi} i(F, z) .
$$

Démonstration. - On peut plonger dans $\mathbf{R}^{2}$ l'anneau $A$, défini par recollement des copies du compactifié, par une application $h$ préservant l'orientation, envoyant $U_{K}$ sur $\mathbf{R}^{2} \backslash \overline{\mathbf{D}}$ et $\sigma\left(U_{K}\right)$ sur $\mathbf{D} \backslash\{0\}$. On note alors $\Gamma_{1}$ l'image de $\partial U$ et $\Gamma_{2}$ l'image de $\sigma(\partial U)$. L'homéomorphisme $G=h \circ F \circ h^{-1}$ est alors défini sur un voisinage de l'anneau fermé délimité par $\Gamma_{1}$ et $\Gamma_{2}$. On a

$$
i\left(G, \Gamma_{1}\right)=i(f, \partial U)
$$

et

$$
i\left(G, \Gamma_{2}\right)=2-i(f, \partial U)
$$

cette dernière relation étant conséquence du fait que la caractéristique d'Euler de la sphère $S^{2}$ est 2. La proposition est alors une conséquence des égalités

$$
\begin{aligned}
\sum_{z \in \operatorname{Fix}(\varphi)} i(F, z)=\sum_{z^{\prime} \in h(\operatorname{Fix}(\varphi))} i\left(G, z^{\prime}\right) & =i\left(G, \Gamma_{1}\right)-i\left(G, \Gamma_{2}\right) \\
& =i(f, \partial U)-(2-i(f, \partial U)) .
\end{aligned}
$$


Remarque. - Si $\varphi$ est un éclatement de $K \in \mathcal{K}$ et si $z \in \operatorname{Fix}(\varphi)$ est un point fixe isolé du symétrisé $F$, c'est un puits, une source ou un point semi-stable de $\varphi$ : dans les deux premiers cas, l'indice $i(F, z)$ est impair, dans le troisième cas il est pair. $\mathrm{Si} \varphi$ est fini et si tous les points fixes de $\varphi$ sont des points isolés de $\operatorname{Fix}(F)$, il y a un nombre identique de puits et de sources de $\varphi:$ l'entier

$$
\sum_{z \in \operatorname{Fix} \varphi} i(F, z)
$$

est bien pair. Il existe alors un voisinage $U \in \mathcal{D}$ de $K$ tel que $\bar{U} \backslash K$ n'a pas de point fixe et on a

$$
i(f, K)=i(f, \partial U)=1+\frac{1}{2} \sum_{z \in \operatorname{Fix} \varphi} i(F, z) .
$$

\section{2. Étude des points fixes d'un éclatement}

Commençons par montrer le résultat suivant.

Proposition 8.2. - Soit $\varphi$ un éclatement de $K \in \mathcal{K}$. Tout point $z \in \operatorname{Fix}(\varphi)$, qui est un point fixe isolé du symétrisé $F$, est non accumulé.

Démonstration. - Soit $z \in \operatorname{Fix}(\varphi)$ un point fixe isolé de $F$. Fixons deux domaines simplement connexes $V$ et $V^{\prime}$ de $U_{K}$, avec $V^{\prime} \cup f\left(V^{\prime}\right) \subset V$, assez proches de $z$ dans l'éclatement $\varphi$ pour ne contenir aucun point fixe et dont les frontières dans l'éclatement sont des courbes de Jordan contenant un voisinage de $z$ dans $S^{1}$ (en d'autres termes la moitié de "petits disques" de $A$ centrés en $z$ ).

On reprend maintenant la démonstration de la proposition 3.1 en changeant quelques détails. On peut toujours supposer $f$ définie sur $\mathbf{R}^{2}$. On note cette fois-ci $X=\operatorname{Fix}(f)$ et $U$ la composante connexe de $\mathbf{R}^{2} \backslash X$ qui contient $V$; celle-ci est invariante car $f(V) \cap V \neq \emptyset$. On note alors $\pi: \widetilde{U} \rightarrow U$ le revêtement universel de $U$; on fixe une composante connexe $V_{0}$ de $\pi^{-1}(V)$, qui est homéomorphe à $V$ puisque $V$ est simplement connexe; on note $V_{0}^{\prime}$ la composante connexe de $\pi^{-1}\left(V^{\prime}\right)$ contenue dans $V_{0}$; enfin on considère le relèvement $\tilde{f}$ de $f_{\mid U}$ tel que $\tilde{f}\left(V_{0}^{\prime}\right) \subset V_{0}$. Si $V^{\prime}$ contient une orbite périodique $O$, l'orbite par $\tilde{f}$ de tout élément de $\pi^{-1}(O) \cap V_{0}^{\prime}$ doit rester dans $V_{0}^{\prime}$ et sera donc périodique. Ceci est impossible car $\tilde{f}$ est un homéomorphisme du plan sans point fixe et préservant l'orientation. Toute orbite périodique de $F$ proche de $z$ doit être située du même côté de $S^{1}$ et peut donc être choisie dans $V^{\prime}$ si le point $z$ est accumulé, cette situation est donc impossible.

Proposition 8.3. - Soit $\varphi$ un éclatement de $K \in \mathcal{K}$. Pour tout point fixe $z$ de $\varphi$ qui est un point fixe isolé de type selle du symétrisé $F$, la suite $i\left(F^{k}, z\right)_{k \geqslant 1}$ est bien définie et constante, égale à un entier $s \leqslant 0$. Si, de plus, $z$ est un puits ou une source de $\varphi$, on a $s \leqslant-1$.

Démonstration. - La proposition 8.2 nous dit que cette suite est bien définie. Il reste alors à montrer que l'entier $q$ qui apparaît dans la proposition 3.3 est égal à 1 . Or le cas où $q \geqslant 2$ n'est possible qu'en présence d'une certaine rotation au voisinage du point $z$ (par exemple pour un point fixe de type selle avec réflexion, voir [25]). Ceci ne se produit pas dans notre cas, à cause de l'invariance de $S^{1}$ et de chacun de ses côtés. On peut se référer à la démonstration originale de la proposition 3.3 pour obtenir le résultat. On peut également utiliser l'artifice suivant.

On considère un petit domaine de Jordan $\Delta$ de $A$ centré en $z$, invariant par $\sigma$, ne contenant aucune orbite périodique. On peut définir un homéomorphisme local $F_{0}$ défini sur $U$, commutant avec $\sigma$, coïncidant avec $F$ sur $S^{1} \cap \Delta$, et conjuguée sur $\Delta$ au modèle standard d'une selle 
dégénérée possédant $r$ demi-variétés stables fixes alternant avec $r$ demi-variétés instables fixes. On peut alors considérer l'homéomorphisme local $F_{1}$ défini sur $\Delta$, égal à $F$ d'un côté de $S^{1}$ et à $F_{0}$ de l'autre, ainsi que l'homéomorphisme $F_{2}=\sigma \circ F_{1} \circ \sigma^{-1}$ qui a des propriétés analogues. Il est facile de voir que $z$ est un point fixe de type selle non accumulé pour chacune des applications $F_{0}, F_{1}$ et $F_{2}$, plus précisément que $\Delta$ est isolant et ne contient pas d'orbites périodiques. On peut appliquer la proposition 3.3 à chacune de ces applications. Si on choisit $r$ assez grand, chacun des indices

$$
i\left(F_{0}, z\right), \quad i\left(F_{1}, z\right), \quad i\left(F_{2}, z\right)
$$

sera négatif et chacune des suites

$$
\left(i\left(F_{0}^{k}, z\right)\right)_{k \geqslant 1}, \quad\left(i\left(F_{1}^{k}, z\right)\right)_{k \geqslant 1}, \quad\left(i\left(F_{2}^{k}, z\right)\right)_{k \geqslant 1}
$$

sera donc constante. On en déduit que la suite $\left(i\left(F^{k}, z\right)\right)_{k \geqslant 1}$ est constante en utilisant l'égalité

$$
i\left(F^{k}, z\right)+i\left(F_{0}^{k}, z\right)=i\left(F_{1}^{k}, z\right)+i\left(F_{2}^{k}, z\right)
$$

qui se démontre en calculant l'indice sur le bord d'un petit domaine de Jordan $\Delta^{\prime} \subset \Delta$ invariant $\operatorname{par} \sigma$.

\section{Application à la dynamique locale au voisinage d'un point fixe}

\subsection{Indice d'un point fixe isolé dans le cas conservatif}

Le paragraphe précédent permet de retrouver le résultat suivant de Pelikan et Slaminka [31], montré auparavant pour les difféomorphismes par N.A. Nikishin [29] et C.P. Simon [34].

THÉORÈME 9.1. - Si $f: W \rightarrow W^{\prime}$ préserve une mesure borélienne chargeant les ouverts et si 0 est un point fixe isolé, alors $i(f, 0) \leqslant 1$.

Démonstration. - Grâce aux résultats du paragraphe 3, il suffit de démontrer le théorème dans le cas où $z$ est indifférent et non accumulé. On considère alors un domaine de Jordan $U_{0}$, dont l'adhérence est contenue dans $W$ et ne contient aucune orbite périodique, tel que pour tout domaine de Jordan $U \subset U_{0}$, la composante connexe $K$ de $\bigcap_{k \in \mathbf{Z}} f^{-k}(\bar{U})$ qui contient 0 rencontre $\partial U$. On choisit $U$ vérifiant $\bar{U} \subset U_{0}$. Grâce à la proposition 7.2, on peut trouver un facteur non indifférent de l'éclatement $\varphi_{K}$ défini par les bouts premiers. Puisque $f$ préserve une mesure chargeant les ouverts, aucun des points fixes (s'il y en a) de $\varphi$ n'est un point fixe dissipatif du symétrisé $F$ : c'est donc un point-selle. En particulier, on a $i(F, z) \leqslant 0$ et on peut conclure par la proposition 8.1 puisque

$$
i(f, z)=i\left(f, \partial U_{0}\right) \leqslant 1 .
$$

\subsection{Dynamique au voisinage d'un point fixe d'indice $\geqslant 2$}

On va préciser le théorème 9.1. On peut trouver une version plus faible du résultat qui suit dans [23], où l'on montre que l'une au moins des assertions (i) ou (ii) est vérifiée sous les hypothèses du théorème.

THÉORÈme 9.2. - On suppose que 0 est un point fixe isolé de $f: W \rightarrow W^{\prime}$ et que $i(f, 0) \geqslant 2$. On a alors les deux propriétés suivantes: 
(i) il existe un domaine $V \subset W$ tel que la suite $\left(f^{n}(V)\right)_{n \geqslant 0}$ est bien définie et formée de domaines disjoints deux à deux de $W$;

(ii) il existe un domaine $V^{\prime} \subset W^{\prime}$ tel que la suite $\left(f^{n}\left(V^{\prime}\right)\right)_{n \leqslant 0}$ est bien définie et formée de domaines disjoints deux à deux de $W^{\prime}$.

Démonstration. - Sous les hypothèses du théorème, le point fixe est indifférent et non accumulé. On reprend alors la démonstration précédente en gardant les mêmes notations. On sait alors que l'on a

$$
\sum_{z \in \operatorname{Fix}(\varphi)} i(F, z)=2(i(f, 0)-1) \geqslant 2 .
$$

L'application $\varphi$ a donc des points fixes, qui sont des points fixes non indifférents de $F$. Notons $A_{1}$ l'ensemble des puits de $\varphi, A_{2}$ l'ensemble des sources de $\varphi$ et $A_{3}$ l'ensemble des point semistables. Chaque point fixe $z \in A_{3}$ est un point-selle de $F$ puisqu'il n'est pas dissipatif : on a donc $i(F, z) \leqslant 0$. On en déduit que l'on a

$$
\sum_{z \in A_{1}} i(F, z)+\sum_{z \in A_{2}} i(F, z) \geqslant 2
$$

Pour montrer le théorème, il suffit de montrer que l'un des puits et l'une des sources de $\varphi$ sont un point fixe dissipatif de $F$. Ceci est une conséquence de l'égalité ci-dessus et des remarques suivantes :

- si $z \in A_{1} \cup A_{2}$ est dissipatif, alors $i(F, z)=1$;

- si $z \in A_{1} \cup A_{2}$ est un point selle, alors $i(F, z) \leqslant-1$;

- les ensembles $A_{1}$ et $A_{2}$ ont même cardinal.

\subsection{Un résultat de $M$. Brown}

On peut retrouver le résultat suivant de M. Brown [6].

THÉORÈME 9.3. - Si 0 est un point fixe isolé de $f: W \rightarrow W^{\prime}$ et si $i(f, 0) \neq 1$, alors l'indice $i\left(f^{k}, 0\right)$ est bien défini pour tout $k \neq 0$ et indépendant de $k$.

Démonstration. - On sait, grâce à la proposition 3.1 que 0 n'est pas accumulé et grâce à la proposition 3.2 que 0 n'est pas dissipatif. Si 0 est un point selle, le théorème est une conséquence de la proposition 3.3. Si 0 est indifférent, on reprend la démonstration du théorème 9.1 en gardant les mêmes notations. Pour chaque point fixe $z$ de $\varphi$, la suite $\left(i\left(F^{k}, z\right)\right)_{k \geqslant 1}$ est constante, que $z$ soit un point fixe dissipatif de $F$ ou un point-selle. On applique alors la proposition 8.1.

\subsection{Détermination de la suite $i\left(f^{k}, 0\right)_{k \geqslant 1}$}

En fait, on a démontré le résultat suivant :

THÉORÈME 9.4. - Si 0 est un point fixe indifférent non accumulé de $f$, alors il existe $q \geqslant 1$ et $r \in \mathbf{Z}$ tels que pour tout $k \in \mathbf{Z}$ :

$$
\begin{cases}i\left(f^{k}, 0\right)=1 & \text { si } k \notin q \mathbf{Z}, \\ i\left(f^{k}, 0\right)=r & \text { si } k \in q \mathbf{Z} .\end{cases}
$$

L'entier q est le dénominateur du nombre de rotation de 0 , écrit sous forme irréductible, si celuici est rationnel; il est égal à 1 si celui-ci est irrationnel. 


\subsection{Un résultat dans le cas conservatif}

Terminons ce paragraphe par un résultat valable dans le cas conservatif. On suppose que $f: W \rightarrow W^{\prime}$ préserve une mesure borélienne chargeant les ouverts ou plus généralement que la conclusion du théorème 9.2 n'est pas vérifiée. On suppose de plus que 0 est indifférent non accumulé. On considère un domaine de Jordan $U_{0}$, dont l'adhérence est contenue dans $W$ et ne contient aucune orbite périodique, tel que pour tout domaine de Jordan $U \subset U_{0}$, la composante connexe $K$ de $\bigcap_{k \in \mathbf{Z}} f^{-k}(\bar{U})$ qui contient 0 rencontre $\partial U$. On note alors $\mathcal{K}_{0}$ (resp. $\mathcal{K}_{0}^{\prime}$ ) l'ensemble des éléments de $\mathcal{K}$ inclus dans $\bar{U}_{0}$ (resp. dans $U_{0}$ ) et on définit

$$
\mathcal{K}_{0}^{*}=\mathcal{K}_{0} \backslash\{\{0\}\}, \quad \mathcal{K}_{0}^{* *}=\mathcal{K}_{0}^{\prime} \backslash\{\{0\}\} .
$$

Proposition 9.5. - Si la suite $\left(i\left(f^{k}, z\right)\right)_{k \geqslant 1}$ est constante égale à 1 , trois cas sont possibles :

(i) le nombre de rotation $\rho \in \mathbf{T}^{1}$ de 0 est irrationnel, c'est le nombre de rotation de tout éclatement de $K \in \mathcal{K}_{0}^{\prime}$;

(ii) le nombre de rotation $\rho$ est rationnel, tout éclatement de $K \in \mathcal{K}_{0}^{\prime}$ a un nombre de rotation égal à $\rho$ et est semi-stable à droite;

(iii) le nombre de rotation $\rho$ est rationnel, tout éclatement de $K \in \mathcal{K}_{0}^{\prime}$ a un nombre de rotation égal à $\rho$ et est semi-stable à gauche.

Démonstration. - Par définition, nous savons que $\rho$ est le nombre de rotation commun des $\varphi_{K}, K \in \mathcal{K}_{0}^{*}$. Supposons d'abord que ce ce nombre est nul et démontrons la série d'assertions suivantes.

(a) Les homéomorphismes $\varphi_{K}, K \in \mathcal{K}_{0}^{*}$, ne sont jamais égaux à l'identité.

C'est une conséquence immédiate du lemme 5.2.

(b) L'ensemble des compacts $K \in \mathcal{K}_{0}^{*}$, avec $\varphi_{K}$ semi-stable à droite (resp. semi-stable à gauche), est fermé dans $\mathcal{K}_{0}^{*}$.

C'est un corollaire immédiat de la proposition 5.3.

(c) Pour tout $U \in \mathcal{D}$ inclus dans $U_{0}$, l'homéomorphisme $\varphi_{K}$ est semi-stable, où $K$ est la composante connexe de $\bigcap_{k \in \mathbf{Z}} f^{-k}(\bar{U})$ qui contient 0 .

Si ce n'est pas le cas, nous pouvons trouver un facteur fini $\varphi$ de $\varphi_{K}$, plus fin que le facteur défini dans la proposition 7.1 (et donc non indifférent) qui admet au moins un puits et une source (dans $S^{1}$ ). Il suffit d'ajouter aux intervalles $I_{k}$ définis dans cette proposition, dans le cas où la dynamique est toujours dans le même sens sur ces intervalles, un autre intervalle de $S^{1} \backslash \operatorname{Fix}\left(\varphi_{K}\right)$ où la dynamique est dans le sens contraire, puis de prendre le facteur naturellement défini. Puisque les puits et les sources de $\varphi$ ne sont pas des points fixes dissipatifs de $F$ mais des pointsselles d'indice de Lefschetz au plus égal à -1 , nous en déduisons grâce à la proposition 8.1 que $i(f, 0) \leqslant 0$, ce qui est impossible.

(d) Pour tout $K \in \mathcal{K}_{0}^{* *}$, l'homéomorphisme $\varphi_{K}$ est semi-stable.

C'est une conséquence des assertions (b) et (c), ainsi que du lemme 6.1 qui énonce que tout compact plein $K \in \mathcal{K}_{0}^{\prime}$ est limite d'une suite strictement décroissante de compacts du type décrit dans (c).

(e) L'ensemble des compacts $K \in \mathcal{K}_{0}^{\prime *}$, avec $\varphi_{K}$ semi-stable à droite (resp. semi-stable à gauche), est ouvert dans $\mathcal{K}_{0}^{\prime *}$.

C'est une conséquence des assertions (a), (b) et (d).

(f) Les homéomorphismes $\varphi_{K}, K \in \mathcal{K}_{0}^{*}$, sont tous semi-stables à droites ou tous semi-stables à gauche.

Notons $K_{0}$ la composante connexe de $\bigcap_{k \in \mathbf{Z}} f^{-k}\left(\bar{U}_{0}\right)$ et supposons que $\varphi_{K_{0}}$ est semi-stable à droite. S'il existe $K \in \mathcal{K}_{0}^{*}$ tel que $\varphi_{K}$ soit semi-stable à gauche, nous pouvons considérer un 
élément maximal $K_{1}$, dans $\mathcal{K}_{0}^{*}$ vérifiant cette propriété. De la remarque qui suit la proposition 5.3 et du fait que $f$ n'a pas de point fixe sur $\partial U_{0}$, on en déduit que $K_{1}$ ne peut pas rencontrer $\partial U_{0}$ et appartient donc à $\mathcal{K}_{0}^{\prime *}$. Il est plein et limite d'une suite strictement décroissante d'éléments de $\mathcal{K}_{0}^{\prime *}$. La contradiction provient de l'assertion (e) et de la maximalité de $K_{1}$.

On a une situation analogue si le nombre de rotation est rationnel. Pour montrer la proposition, on considère maintenant un éclatement $\varphi$ de $K \in \mathcal{K}_{0}^{\prime}$. On peut toujours supposer que $K$ est plein et considérer une suite strictement décroissante $\left(K_{n}\right)_{n \geqslant 0}$ d'éléments de $\mathcal{K}_{0}$ convergeant vers $K$. Le corollaire 5.4 et la construction faite dans la proposition 6.1 montre que la suite des nombres de rotation $\left(\rho_{K_{n}}\right)_{n \geqslant 0}$ converge vers le nombre de rotation de $\varphi$ et que celui-ci est donc égal à $\rho$. Si ce nombre est rationnel et si chaque $\varphi_{K_{n}}$ est semi-stable à droite (resp. à gauche) il en sera de même de $\varphi$. On ne peut pas affirmer dans cette démonstration que $\varphi$ est différent de l'identité car le lemme 5.2 n'est démontré que pour les éclatements par bouts premiers.

Remarques. - La proposition 9.4 nous dit en particulier que si $K \in \mathcal{K}$ contient $z$ et admet un voisinage $U_{1}$ qui ne contient aucun point périodique autre que $z$, alors $\rho_{K}=\rho$ et la dynamique de $\varphi_{K}$ est semi-stable à droite dans le cas (i) et semi-stable à gauche dans le cas (ii). En effet, d'après la remarque qui suit la proposition 3.3, aucun domaine de Jordan $U \subset U_{1}$ contenant 0 n'est isolant et par l'argument vu au paragraphe 2 sur les points indifférents, la composante connexe de $\bigcap_{k \in \mathbf{Z}} f^{-k}(\bar{U})$ rencontre $\partial U$. On peut donc appliquer la proposition 9.4 avec $U_{0}=U_{1}$.

Il n'est pas évident que les situations (ii) et (iii) soient elles-mêmes possibles. Si on peut montrer qu'elles sont impossibles, on obtiendra une preuve bien plus simple des résultats des paragraphes 11 et 12 .

\section{Rappels sur les homéomorphismes sans points errants de l'anneau compact}

Nous commencerons dans ce paragraphe par rappeler certains résultats sur les homéomorphismes conservatifs de l'anneau compact, dus à J. Franks, dont nous aurons besoin plus tard. Nous rajouterons un énoncé qui nous sera nécessaire.

On note

$$
\begin{aligned}
\pi: \mathbf{R} \times[0,1] & \rightarrow \mathbf{T}^{1} \times[0,1], \\
(x, y) & \mapsto(x+\mathbf{Z}, y)
\end{aligned}
$$

le revêtement universel de l'anneau $\mathbf{T}^{1} \times[0,1]$ et

$$
\begin{aligned}
T: \mathbf{R} \times[0,1] & \rightarrow \mathbf{R} \times[0,1], \\
(x, y) & \mapsto(x+1, y)
\end{aligned}
$$

la transformation de recouvrement.

\subsection{Ensemble de rotation}

On se donne un homéomorphisme $f$ de $\mathbf{T}^{1} \times[0,1]$ homotope à l'identité et un relèvement $\tilde{f}$ de $f$ au revêtement universel. Si $O$ est une orbite périodique de $f$ de période $q$ et si $z \in \pi^{-1}(O)$, il existe un entier $p \in \mathbf{Z}$, indépendant de $z$, tel que $f^{q}(z)=T^{p}(z)$. Le nombre rationnel $p / q$ est par définition le nombre de rotation de $O$. Plus généralement si $\mu$ est une mesure borélienne de probabilité invariante par $f$, son nombre de rotation (pour le relèvement $\tilde{f}$ ) est

$$
\tilde{\rho}(\mu)=\int_{\mathbf{T}^{1} \times[0,1]} p_{1} \circ \tilde{f}-p_{1} d \mu,
$$


où $p_{1}:(x, y) \mapsto x$ est la première projection. L'ensemble de rotation de $\tilde{f}$ est, par définition, l'ensemble des nombres de rotation des mesures boréliennes de probabilité invariantes de $f$, c'est un segment.

\subsection{Disques positifs et négatifs}

Un disque positif (resp. un disque négatif) de $\tilde{f}$ est un domaine simplement connexe $V$ de $\mathbf{R} \times] 0,1[$ vérifiant

$$
\tilde{f}(V) \cap V=\emptyset
$$

et

$$
\tilde{f}^{q}(V) \cap T^{p}(V) \neq \emptyset
$$

pour des entiers $q>0$ et $p>0$ (resp. $p<0$ ).

\subsection{Critères d'existence d'orbites périodiques}

Le lemme crucial, dans cette étude, est la proposition 10.1 due à J. Franks, qui est conséquence du théorème d'indice de Brouwer (Proposition 1.1). On utilisera également les corollaires 10.2 et 10.3 et la proposition 10.4 (voir Franks [13-15]) :

Proposition 10.1. - On suppose que $f$ n'a pas de point errant. Si $\tilde{f}$ admet un disque positif et un disque négatif, alors $\tilde{f}$ a un point fixe.

Appliquant ce lemme aux applications $f^{q} \circ T^{-p}$, on obtient :

COROLlaire 10.2. - Si f n'a pas de points errants, l'ensemble des nombres de rotation des orbites périodiques de $f$ est un intervalle de $\mathbf{Q}$.

En utilisant, de plus, le théorème ergodique de Birkhoff, on a :

COROLlaire 10.3. - On suppose que $f$ n'a pas de point errant. Tout nombre rationnel $\tilde{\rho}$, appartenant à l'intérieur de l'ensemble de rotation de $\tilde{f}$ est nombre de rotation d'une orbite périodique de période q de $f$, l'entier q étant le dénominateur de $\tilde{\rho}$, écrit sous forme irréductible.

Énonçons maintenant la variante suivante du théorème de Poincaré-Birkhoff, due également à Franks :

Proposition 10.4. - On suppose que toute courbe non homotope à zéro de $\mathbf{T}^{1} \times[0,1[$ rencontre son image par $f$. Tout nombre rationnel, compris entre les nombres de rotation définis sur les deux bords par le relèvement $\tilde{f}$ est nombre de rotation d'une orbite périodique de $f$.

\subsection{Un nouveau critère d'existence d'orbites périodiques}

Proposition 10.5. - On suppose que $f$ n'a pas de point errant. Si $\tilde{f}$ a un point fixe et s'il existe un arc simple joignant le bord $\mathbf{R} \times\{0\}$ au bord $\mathbf{R} \times\{1\}$ dont l'image $\Gamma$ vérifie $\tilde{f}(\Gamma) \cap \Gamma=\emptyset$, alors l'ensemble des nombres de rotation des orbites périodiques de f est un intervalle non trivial de $\mathbf{Q}$.

Démonstration. - On peut toujours supposer que $\tilde{f}(\Gamma)$ est à droite de $\Gamma$. On considère alors un domaine simplement connexe $V_{0}$ compris entre $\Gamma$ et $f(\Gamma)$. Puisque $f$ n'a pas de point errant, on peut considérer le premier entier strictement positif, noté $q_{0}$, tel que

$$
f^{q_{0}}\left(\pi\left(V_{0}\right)\right) \cap \pi\left(V_{0}\right) \neq \emptyset,
$$


puis le plus petit entier, noté $p_{0} \in \mathbf{Z}$, tel que

$$
\tilde{f}^{q_{0}}\left(V_{0}\right) \cap T^{p_{0}}\left(V_{0}\right) \neq \emptyset .
$$

Le fait que $V_{0}$ soit compris entre $\Gamma$ et $\tilde{f}(\Gamma)$ nous dit que $p_{0} \geqslant 1$. Si l'application $\tilde{f}^{q_{0}} \circ T^{-1}$ a un point fixe, la démonstration est terminée d'après le corollaire 10.2. Dans le cas contraire, on va voir que l'on peut toujours choisir $V_{0}$ pour que l'entier $p_{0}$ soit supérieur ou égal à 2 . Supposons que $p_{0}$ soit égal à 1 , fixons $z \in V_{0} \cap \tilde{f}^{-q_{0}}\left(T\left(V_{0}\right)\right)$ puis un domaine simplement connexe $V_{1} \subset V_{0}$, voisinage de $z$ assez petit pour avoir

$$
\tilde{f}^{q_{0}}\left(V_{1}\right) \cap T\left(V_{1}\right)=\emptyset
$$

et

$$
\tilde{f}^{q_{0}}\left(V_{1}\right) \subset T\left(V_{0}\right),
$$

ce qui est possible puisque $\tilde{f}^{q_{0}}(z) \neq z$. On considère le premier entier strictement positif, noté $q_{1}$, tel que

$$
F^{q_{1}}\left(\pi\left(V_{1}\right)\right) \cap \pi\left(V_{1}\right) \neq \emptyset,
$$

puis le plus petit entier, noté $p_{1}$, tel que

$$
f^{q_{1}}\left(V_{1}\right) \cap T^{p_{1}}\left(V_{1}\right) \neq \emptyset .
$$

On voit immédiatement que l'on a $q_{1} \geqslant q_{0}$ et même $q_{1} \geqslant 2 q_{0}$. De l'inclusion

$$
\tilde{f}^{q_{1}}\left(V_{1}\right) \subset \tilde{f}^{q_{1}-q_{0}}\left(T\left(V_{0}\right)\right)
$$

on déduit que l'on a

$$
\tilde{f}^{q_{1}}\left(V_{1}\right) \cap T^{p}\left(V_{1}\right)=\emptyset
$$

si $p \leqslant 1$. Ainsi on a $p_{1} \geqslant 2$.

Le domaine $V_{1}$ est un disque positif du relèvement $\tilde{g}=\tilde{f}^{q_{1}} \circ T^{-1}$ de $f^{q_{1}}$. On obtient un disque négatif de $g$ en choisissant un domaine de Jordan, voisinage assez petit du point fixe $z$ de $\tilde{f}$ si celui-ci n'est pas sur le bord de l'anneau; en choisissant un domaine de Jordan assez proche de $z$ et dont la frontière contient un voisinage de $z$ dans le bord de l'anneau, si ce point fixe est sur le bord de l'anneau. Comme $g$ n'a pas de point errant, on en déduit que $\tilde{g}$ a un point fixe. Le corollaire 10.2 nous dit que l'ensemble des nombres de rotation des orbites périodiques de $f$ contient l'intervalle $\left[0,1 / q_{1}\right]$.

\section{Application aux homéomorphismes conservatifs de la sphère}

On va montrer dans ce paragraphe le théorème suivant, qui généralise un résultat de $\mathrm{M}$. Handel [18] (cas où $f$ est topologiquement transitif) ainsi qu'un résultat de J. Franks [16] (cas où $f$ préserve une mesure de probabilité chargeant les ouverts).

THÉORÈME 11.1. - Si $f: S^{2} \rightarrow S^{2}$ a au moins trois points fixes et n'a pas de point errant, alors $f$ a une infinité d'orbites périodiques.

Commençons par quelques compléments sur les résultats obtenus jusqu'au paragraphe 9. 
Soit $f$ un homéomorphisme préservant l'orientation d'une surface orientable $M$ et $K$ un continu plein et contractile dans $M$. On dira que $K$ est un continu accumulé, non accumulé, indifférent, dissipatif, de type selle si le point fixe $\{K\}$ de l'homéomorphisme $\hat{f}$ défini naturellement sur la surface $\widehat{M}$ obtenue en identifiant $K$ à un point, a la propriété correspondante. On peut définir également la compactification par bouts premiers de $K$, ainsi que l'élément $\rho_{K}=\rho_{\widetilde{K}} \in \mathbf{T}^{1}$ et l'homéomorphisme $\varphi_{K}=\varphi_{\widetilde{K}}$ de $S^{1}$. Les résultats locaux montrés pour les points fixes se généralisent immédiatement à ces ensembles.

Démonstration du théorème 11.1. - On suppose que $f$ vérifie les hypothèses du théorème et a un nombre fini d'orbites périodiques. Quitte à prendre un itéré pair assez grand de $f$, on peut supposer que $f$ préserve l'orientation et que tous les points périodiques sont des points fixes. Ces points n'étant pas accumulés, on peut utiliser la proposition 3.3 et le théorème 9.4 : quitte à prendre un itéré plus grand de $f$, on peut toujours supposer que, pour tout point fixe $z$, la suite $i\left(f^{k}, z\right)_{k \geqslant 1}$ est constante. Le théorème 9.2 nous dit que chaque indice $i(f, z)$ est inférieur ou égal à 1 : on en déduit, par la formule de Lefschetz, qu'il existe au moins deux points fixes $z_{0}$ et $z_{1}$ tels que

$$
i\left(f, z_{0}\right)=i\left(f, z_{1}\right)=1 .
$$

Puisque $f$ n'a pas de points errants, ces points ne sont pas dissipatifs. Comme ils ne sont pas accumulés et comme les indices des itérés sont tous égaux à 1, ce ne sont pas des points-selles (Proposition 3.3) : ce sont donc des points indifférents non accumulés, en particulier on peut définir leurs nombres de rotation, éléments de $\mathbf{T}^{1}$, notés respectivement $\rho_{0}$ et $\rho_{1}$.

Supposons d'abord que l'un des nombres de rotation est non nul. On considère deux continus pleins disjoints $K_{0}$ et $K_{1}$, invariants par $f$, contenant respectivement $z_{0}$ et $z_{1}$ et ne se réduisant pas à ces points, suffisamment proches, respectivement de $z_{0}$ et $z_{1}$, pour ne contenir aucun autre point fixe de $f$ et pour que les nombres de rotation définis par les bouts premiers sur $S^{2} \backslash K_{0}$ et $S^{2} \backslash K_{1}$ soient $\rho_{0}$ et $\rho_{1}$. La restriction de $f$ à l'anneau ouvert $S^{2} \backslash\left(K_{0} \cup K_{1}\right)$ se prolonge en un homéomorphisme du compactifié obtenu en ajoutant les cercles des bouts premiers en chaque continu. L'homéomorphisme obtenu n'a pas de point errant, il possède un point fixe (puisque $f$ a au moins trois points fixes!) et l'un des nombres $\rho_{0}$ ou $\rho_{1}$ n'est pas nul. Ceci contredit le corollaire 10.2.

Supposons maintenant que $\rho_{0}$ et $\rho_{1}$ sont nuls. On sait que l'homéomorphisme défini sur le cercle des bouts premiers en $K_{0}$ n'est pas l'identité. On considère un éclatement $\varphi$ de $K_{0}$, facteur de l'éclatement par bouts premiers, qui possède exactement un point fixe $z_{2}$. La restriction de $f$ à $S^{2} \backslash K_{0}$ se prolonge au compactifié défini par l'éclatement. On note $F$ le symétrisé de $f$ par rapport à $S^{1}$, qui est donc défini sur une sphère topologique $\Sigma$. On considère un système de coordonnées polaires $(\theta, r) \in \mathbf{T}^{1} \times[-1,1]$ défini sur un voisinage de $S^{1}$ ne contenant aucun autre point fixe que $z_{2}$, tel que :

- le cercle $S^{1}$ est le cercle d'équation $r=0$;

- un point $z=(\theta, r)$ appartient à $S^{2}-K_{0}$ si et seulement si $r>0$;

- on a $z_{2}=(0,0)$

- on a $\sigma(\theta, r)=(\theta,-r)$, où $\sigma$ est l'involution naturellement définie sur $\Sigma$.

Fixons $\varepsilon_{0}>0$ assez petit pour que l'intersection de l'arc $] \varepsilon_{0}, 1-\varepsilon_{0}[\times\{0\}$ et de son image par $F$ soit non vide. Fixons ensuite $\varepsilon_{1}>0$ assez petit pour que chaque segment $\{\theta\} \times\left[-\varepsilon_{1}, \varepsilon_{1}\right]$ soit disjoint de son image si $\theta \in\left[\varepsilon_{0}, 1-\varepsilon_{0}\right]$. Considérons les domaines de Jordan

$$
U=] \varepsilon_{0}, 1-\varepsilon_{0}[\times]-\varepsilon_{1}, \varepsilon_{1}[
$$


et

$$
V=\Sigma \backslash \bar{U}
$$

et posons

$$
X=\bigcap_{k \in \mathbf{Z}} f^{-k}(\bar{V})
$$

Montrons maintenant les assertions suivantes :

(i) L'ensemble $X$ a un nombre fini de composantes connexe fixes.

En effet, chaque composante connexe fixe de $K$ est pleine, d'après le choix de $\varepsilon_{0}$, et contient au moins un point fixe d'après le théorème de Cartwright-Littlewood [9] (rappelons en passant que ce théorème est une conséquence directe du théorème de translation de Brouwer, voir [4]). Remarquons également que chaque point fixe de $F$ est contenu dans une composante connexe fixe de $X$.

(ii) Pour chaque composante connexe fixe $K$ de $X$, on a $i(F, K) \leqslant 1$.

En effet, $F$ n'a pas de point errant et on peut appliquer le théorème 9.2.

(iii) Pour chaque composante connexe fixe de $X$, la suite $(i(F, K))_{k \geqslant 1}$ est constante.

En effet, on a

$$
i\left(F^{k}, K\right)=\sum_{z \in \operatorname{Fix}(F) \cap K} i\left(F^{k}, z\right)
$$

et on sait que la suite $\left(i\left(F^{k}, z\right)\right)_{k \geqslant 1}$ est constante pour tout point fixe $z$ de $F$ (même pour $z_{2}$ ).

(iv) Si $K$ est une composante connexe fixe de $X$, il en est de même de $\sigma(K)$ et on a $i(F, K)=i(F, \sigma(K))$.

C'est évident.

(v) Il existe une composante connexe fixe $K_{2}$ de $X$, qui est incluse dans $S^{2} \backslash K_{0}$ et qui vérifie $i\left(F, K_{2}\right)=1$.

$\mathrm{Si}$ on applique la formule de Lefschetz à la sphère obtenue en identifiant chacune des composantes fixes de $X$ à un point, et si on utilise l'assertion (ii), on sait qu'il existe au moins deux composantes fixes d'indice 1 , et donc au moins une qui ne contient pas $z_{2}$. On peut toujours la supposer incluse dans $S^{2} \backslash K_{0}$ d'après (iv).

(vi) La composante $K_{2}$ rencontre la frontière de $V$.

D'après (iii) on sait que le continu $K_{2}$ est indifférent et non accumulé. S'il était contenu dans $V$, on pourrait trouver un continu invariant $K_{3}$ contenant strictement $K_{2}$ et inclus dans $V$, ce qui contredirait le fait que $K_{2}$ est une composante connexe de $X$.
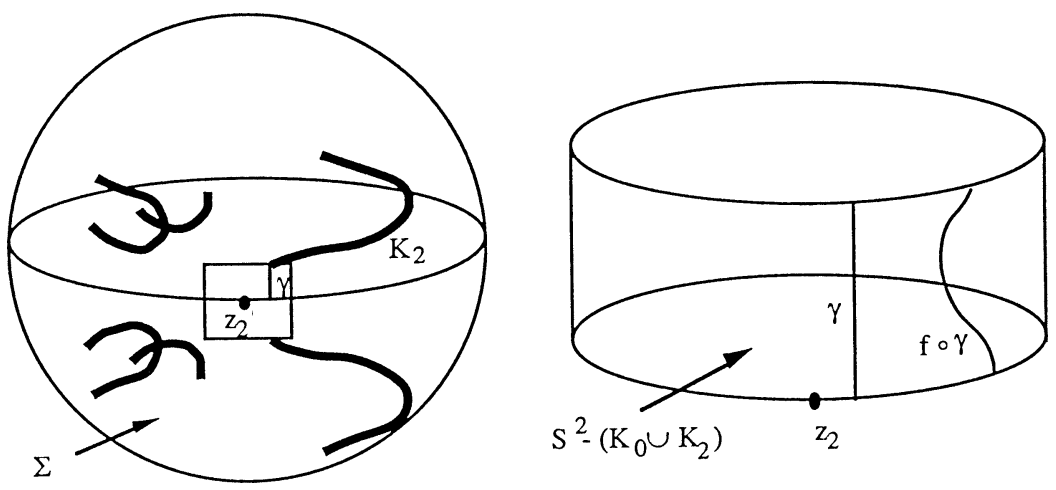

Fig. 5. 
On va maintenant conclure. On peut toujours chosir le point $z=(\theta, r) \in K_{2} \cap \partial V$ de telle façon que l'image de l'arc

$$
\begin{aligned}
\gamma:[0, r[ & \rightarrow S^{2}-K_{0}, \\
t & \mapsto(\theta, t)
\end{aligned}
$$

soit disjoint de $K_{2}$. La partie ouverte annulaire $S^{2} \backslash\left(K_{0} \cup K_{2}\right)$ se compactifie par l'éclatement $\varphi$ en $K_{0}$ et par l'éclatement par bouts premiers en $K_{2}$. La restriction de $F$ à cet anneau se prolonge en un homéomorphisme de l'anneau compact. Il n'a pas de point errant et il a un point fixe (à savoir le point fixe de $\varphi$ ). De plus l' arc $\gamma$ a une limite en $r$ et joint les deux bords de l'anneau. Les $\operatorname{arcs} \gamma$ et $f \circ \gamma$ ont des images disjointes. La contradiction provient de la proposition 10.5.

\section{Application aux homéomorphismes conservatifs du tore}

Nous allons démontrer dans ce paragraphe le résultat suivant.

THÉORÈME 12.1. - Soit $f$ un homéomorphisme du tore $\mathbf{T}^{2}=\mathbf{R}^{2} / \mathbf{Z}^{2}$ homotope à l'identité et $\tilde{f}$ un relèvement de $f \grave{a} \mathbf{R}^{2}$. On suppose que f préserve une mesure de probabilité invariante $\mu$ chargeant les ouverts et ayant un vecteur de rotation

$$
\rho(\mu)=\int_{\mathbf{T}^{2}} \tilde{f}-I d d \mu
$$

égal à 0 . Alors $f$ a une infinité d'orbites périodiques.

Ce résultat était déjà connu dans les cas où $f$ est un difféomorphisme générique : il existe alors une infinité d'orbites périodiques de $f$ qui se relèvent en des orbites périodiques de $\tilde{f}$. C'est un résultat qui s'interprète et se généralise dans le cadre de la géométrie symplectique en dimension supérieure (voir Salamon-Zehnder [32], voir également Hofer-Zehnder [21] et Schwarz [33]). Dans la démonstration qui suit on ne montre pas ce résultat plus fort, bien que dans de nombreux cas que l'on étudiera, les orbites périodiques obtenues auront cette propriété. On peut se demander si on ne peut pas modifier la démonstration de façon à obtenir cette version plus forte du théorème.

Le théorème peut être énoncé pour un homéomorphisme homotope à l'identité $f$ d'un tore abstrait, c'est-à-dire d'une variété topologique $M$ homéomorphe à $\mathbf{T}^{2}$, et pour un relèvement $\tilde{f}$ au revêtement universel de $\widetilde{M}$ de $M$. L'homéomorphisme $f$ préserve alors une mesure borélienne de probabilité chargeant les ouverts et le vecteur de rotation de cette mesure, qui est défini abstraitement par $\tilde{f}$ comme élément du premier groupe d'homologie singulière $H_{1}(M, \mathbf{R})$, est nul. À partir d'un triplet $(f, \tilde{f}, \mu)$ vérifiant les hypothèses du théorème, on peut construire des triplets $\left(f^{\prime}, \tilde{f}^{\prime}, \mu^{\prime}\right)$ vérifiant également les hypothèses de la façon suivante :

(i) On pose $\left(f^{\prime}, \tilde{f}^{\prime}, \mu^{\prime}\right)=\left(f^{q}, \tilde{f}^{q}, \mu\right)$, pour un entier $q \geqslant 1$.

(ii) On suppose que $\left(K_{i}\right)_{1 \leqslant i \leqslant n}$ est une famille finie de continus pleins contractiles invariants de $M$, disjoints deux à deux, tels que chaque composante connexe de $\pi^{-1}\left(K_{i}\right), 1 \leqslant i \leqslant n$, est invariante par $f$, où $\pi: \widetilde{M} \rightarrow M$ est le revêtement universel. On considère l'homéomorphisme $f^{\prime}$ défini sur le tore topologique $M^{\prime}$ obtenu en identifiant chaque $K_{i}$ à un point fixe, on considère le relèvement $\tilde{f}^{\prime}$ au rêvetement universel $\widetilde{M^{\prime}}$ de $M^{\prime}$ obtenu en identifiant chaque composante connexe de $\pi^{-1}\left(K_{i}\right)$ à un point fixe, on note $\mu^{\prime}$ la mesure de probabilité conditionnelle sur $M-\bigcup_{1 \leqslant i \leqslant n} K_{i}$.

Avant d'aborder la démonstration, rappelons le résultat suivant de Franks [15], qui est également une conséquence du théorème d'indice de Brouwer. 
Proposition 12.2. - On suppose que le triplet $(f, \tilde{f}, \mu)$ vérifie les hypothèses du théorème 12.1 et que l'ensemble des points fixes de $f$ qui se relèvent en des points fixes de $\tilde{f}$ est fini. Il existe alors une courbe de Jordan $C \subset \widetilde{M}$ telle que $i(\tilde{f}, C)=1$ et donc un point fixe $z$ de $\tilde{f}$ tel que $i(\tilde{f}, z)=i(f, \pi(z))=1$.

Démonstration du théorème 10.1. - On suppose que le triplet $(f, \tilde{f}, \mu)$ vérifie les hypothèses du théorème et a un nombre fini d'orbites périodiques. On va énoncer un certain nombre de résultats pour aboutir à une contradiction.

LEMME 12.3. - Si $K \subset \mathbf{R}^{2}$ est un continu invariant par $\tilde{f}$ non réduit à un point, le nombre de rotation $\rho_{K}$ défini par les bouts premiers de $U_{K}$ est nul.

Démonstration. - La restriction de $\tilde{f}$ à l'anneau ouvert $U_{K}$ se prolonge en un homéomorphisme du compactifié obtenu en ajoutant le cercle des bouts premiers en $K$ et le cercle des directions à l'infini. Il fixe tous les points du second cercle. De plus, il vérifie la propriété d'intersection énoncée dans la proposition 10.4, puisque $f$ préserve une mesure qui charge les ouverts. Si le nombre de rotation $\rho_{K}$ n'est pas nul, l'ensemble des nombres de rotation des orbites périodiques (pour un relèvement donné) est un intervalle non trivial de $\mathbf{Q}$ : ceci est impossible puisque l'ensemble des périodes possibles est fini.

\section{LEMME 12.4. - Tout continu $K \subset \mathbf{R}^{2}$ invariant par $\tilde{f}$ est plein et d'intérieur vide.}

Démonstration. - Supposons que $K$ ne soit pas plein et considérons une composante connexe bornée $U$ de $\mathbf{R}^{2} \backslash K$. Elle est simplement connexe. Elle est également périodique car $\tilde{f}$ préserve la mesure relevée. Quitte à remplacer $\tilde{f}$ par $\tilde{f}^{q}$, on peut la supposer fixe. Le théorème de translation de Brouwer et le théorème 8.1 nous disent que $U$ contient un point fixe $z$ de $F$ d'indice de Lefschetz 1 . Deux cas sont alors possibles

(i) Il existe un entier $k \geqslant 1$ tel que $i\left(f^{k}, z\right) \neq 1$.

Nous savons par le même raisonnement que $f^{k}$ a un autre point fixe dans $U$. On compactifie $U$ en une sphère en identifiant l'unique bout de $U$ à un point fixe et on obtient un homéomorphisme d'une sphère ayant au moins trois points fixes, un nombre fini d'orbites périodiques et n'ayant pas de point errant : ceci contredit le théorème 11.1.

(ii) La suite $\left(i\left(f^{k}, z\right)\right)_{k \geqslant 1}$ est constante égale à 1 .

Le point $z$, qui est non accumulé, est alors indifférent et admet un nombre de rotation $\rho \in \mathbf{T}^{1}$ qui est nul d'après le lemme 12.3. On considère un continu invariant $K^{\prime} \subset U$ et contenant $z$, proche de $\{z\}$ et distinct de $\{z\}$. On considère un $K^{\prime}$-éclatement, facteur de l'éclatement par bouts premiers, ayant un unique point fixe. On prend le symétrisé de $U \backslash K^{\prime}$ par rapport au cercle de l'éclatement, on ajoute un point fixe à chaque bout de l'anneau obtenu. Là encore on obtient un homéomorphisme d'une sphère ayant au moins trois points fixes, un nombre fini d'orbites périodiques et n'ayant pas de point errant.

Le fait que $K$ est d'intérieur vide se démontre de la même façon. Toute composante connexe de l'intérieur de $K$ est simplement connexe (car $K$ est plein) et périodique.

LEMME 12.5. - Tout continu $K \subset \mathbf{R}^{2}$ invariant par $\tilde{f}$ se plonge injectivement par $\pi$ dans le tore $\mathbf{T}^{2}$.

Démonstration. - Le continu $\pi(K)$ est invariant par $f$, il faut montrer qu'il est contractile. Le théorème de Baire nous dit que la réunion des translatés de $K$ par les translations entières est d'intérieur vide et qu'il en donc ainsi de $\pi(K)$. En particulier $\pi(K)$ n'est pas le tore tout entier. Il suffit donc de démontrer que les composantes connexes $U$ de $\mathbf{T}^{2} \backslash \pi(K)$ ne sont ni simplement connexes, ni annulaires. Toute composante $U$ est périodique et on peut la supposer fixe en remplaçant $f$ par $f^{q}$. Elle ne peut pas être simplement connexe sinon $f$ aura une infinité 
d'orbites périodiques dans $U$ suivant le raisonnement du lemme 12.3. Montrons par l'absurde qu'elle ne peut pas être annulaire.

Considérons un revêtement annulaire intermédiaire

$$
\Pi: A \approx \mathbf{T}^{1} \times \mathbf{R} \rightarrow \mathbf{T}^{2}
$$

tel que $\Pi^{-1}(U)$ soit réunion d'ensembles annulaires disjoints translatés, tous homéomorphes à $U$. On considère deux composantes connexes consécutives $\widetilde{U}_{0}$ et $\widetilde{U}_{1}$ de $\Pi^{-1}(U)$ et deux courbes fermées simples $\Gamma_{0}$ et $\Gamma_{1}$ non homotopes à zéro, contenues respectivement dans $\widetilde{U}_{0}$ et $\widetilde{U}_{1}$. Le relèvement $\hat{f}$ de $f$ à $A$, lui-même relevé par $f$, fixe chaque composante connexe de $\Pi^{-1}(U)$ car le vecteur de rotation est nul. On considère l'ensemble annulaire invariant obtenu comme réunion de $\widetilde{U}_{0}$ de $\widetilde{U}_{1}$ et de l'anneau délimité par $\Gamma_{0}$ et $\Gamma_{1}$. Il contient un point fixe, puisque $f$ admet un point fixe. On compactifie cet anneau ouvert en ajoutant deux points fixes en chaque bout. On a obtenu un homéomorphisme sans point errant admettant un nombre fini de points périodiques et qui a au moins trois points fixes.

Suite de la démonstration du théorème 10.1. - Quitte à prendre un itéré de $f$ on peut supposer que tous les points périodiques de $f$ sont des points fixes et que pour tout point fixe $z$, la suite $\left(i\left(f^{k}, z\right)\right)_{k \geqslant 1}$ est constante. Supposons qu'il existe un continu plein contractile $K$ contenant au moins deux points fixes, la suite $i\left(f^{k}, K\right)_{k \geqslant 1}$ est alors constante. Si on identifie ce compact à un point, on garde les mêmes hypothèses. Ainsi, par une suite finie de telles opérations d'identification, on peut toujours supposer qu'il n'existe aucun continu plein contractile contenant au moins deux points fixes de $f$.

On peut supposer qu'aucune des deux courbes $\mathbf{T}^{1} \times\{0\}$ et $\{0\} \times \mathbf{T}^{1}$ ne contient de point fixe. Il existe donc un réel $\delta>0$ tel que toute boule ouverte de rayon $\delta$ centrée en un point du plan ayant au moins une coordonnée entière est disjointe de son image par $\tilde{f}$. Fixons un entier $N>4 \delta$ et considérons le domaine de Jordan $U=] 0, N\left[^{2}\right.$.

Toute composante connexe fixe de

$$
X=\bigcap_{k \in \mathbf{Z}} \tilde{f}^{-k}(\bar{U})
$$

est pleine, d'intérieur vide et se projette injectivement dans le tore. Ainsi $K$ contient un point fixe ( $d$ 'après Cartwright-Littlewood) et un seul (d'après l'hypothèse faite ci-dessus). D'après la proposition 12.2, on sait qu'il existe un point fixe $z$ de $\tilde{f}$ d'indice 1 . La suite $i\left(\tilde{f}^{k}, z\right)_{k \geqslant 1}$ est constante égale à 1 et le point $z$ est indifférent non accumulé. Son nombre de rotation étant nul, on sait donc qu'il est de type semi-stable à droite ou semi-stable à gauche. Supposons le semistable à droite. En particulier, si $K$ est un continu invariant qui ne contient que $z$ comme point fixe, alors $K$ est indifférent non accumulé et $\varphi_{K}$ est semi-stable à droite, de nombre de rotation 0 .

Il y a exactement $N^{2}$ translatés de $z$ dans $U$. La composante connexe $K\left(z^{\prime}\right)$ de $X$ qui contient chacun d'un translaté $z^{\prime}$ de $z$ est elle-même indifférente non accumulée et rencontre donc $\partial U$. Comme la longueur de $\partial U$ est $4 N$, on peut trouver deux translatés $z_{0}$ et $z_{1}$ et un arc simple

$$
\gamma:] 0,1\left[\rightarrow \mathbf{R}^{2} \backslash K\left(z_{0}\right) \cup K\left(z_{1}\right)\right.
$$

tracé sur $\partial U$, de longueur $\leqslant \delta$ tel que $\gamma$ a une limite dans $K\left(z_{0}\right)$ en 0 et une limite dans $K\left(z_{1}\right)$ en 1 . Cet arc est disjoint de son image par définition de $\delta$. C'est un arc d'accès de $K\left(z_{0}\right)$ et de $K\left(z_{1}\right)$. Si on compactifie $\mathbf{R}^{2} \backslash K\left(z_{0}\right) \cup K\left(z_{1}\right)$ en ajoutant le point à l'infini et les cercles des bouts premiers en $K\left(z_{0}\right)$ et $K\left(z_{1}\right)$, on obtient un homéomorphisme d'un anneau compact et 

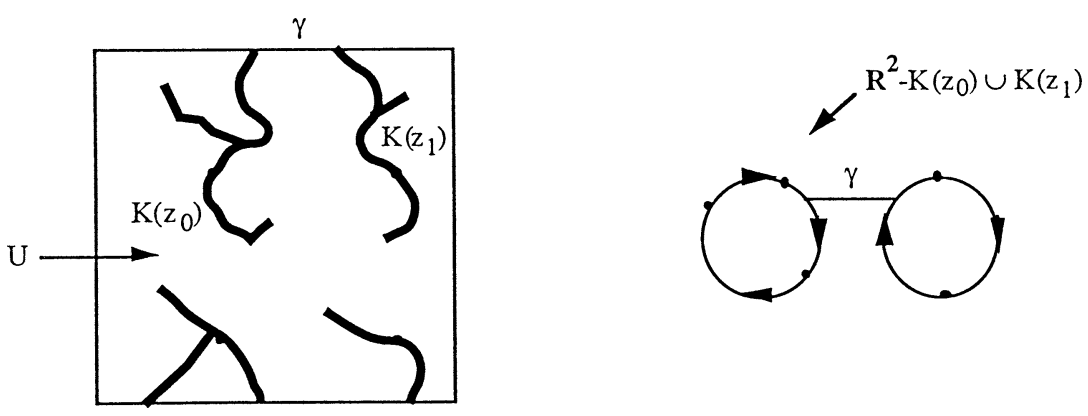

Fig. 6.

un arc joignant un point du bord à un point de l'autre bord qui est disjoint de son image. Ceci contredit le fait que $K\left(z_{0}\right)$ et $K\left(z_{1}\right)$ ont tous deux une dynamique semi-stable à droite.

\section{RÉFÉRENCES}

[1] BiR KhOFF G.-D., An extension of Poincaré's last geometric theorem, Acta Math. 47 (1926) 297-311; in: Collected Math. Papers, Vol. II, Dover, pp. 252-260.

[2] Birkhoff G.-D., Sur quelques courbes fermées remarquables, Bull. Soc. Math. France 80 (1932) 1-26; in: Collected Math. Papers, Vol. II, Dover, pp. 444-461.

[3] Bonino M., Propriétés locales de l'espace des homéomorphismes de Brouwer, Ergodic Theory Dynam. Systems 19 (1999) 1405-1423.

[4] Brouwer L.E.J., Beweis des ebenen Translationssatzes, Math. Ann. 72 (1912) 37-54.

[5] Brown M., A new proof of Brouwer's lemma on translation arcs, Houston J. Math. 10 (1984) 35-41.

[6] Brown M., On the fixed point index of iterates of planar homeomorphisms, Proc. Amer. Math. Soc. 108 (1990) 1109-1114.

[7] CARATHÉOdory C., Über die begrenzung einfach zusammenhangender Gebiete, Math. Ann. 73 (1913) 323-370.

[8] CARTER P.H., An improvment of the Poincaré-Birkhoff theorem, Trans. Amer. Math. Soc. 269 (1982) 285-299.

[9] Cartwright M.L., Littlewood J.C., Some fixed point theorems, Ann. of Math. 54 (1951) 1-37.

[10] FATHI A., An orbit closing proof of Brouwer's lemma on translation arcs, L'Enseignement Math. 33 (1987) 315-322.

[11] FLUCHER M., Fixed points of measure preserving torus homeomorphisms, Manuscripta Math. 68 (1990) 271-293.

[12] FORD L.R., Automorphic Functions, Chelsea Publishing, New York, 1951.

[13] Franks J., Generalizations of the Poincaré-Birkhoff theorem, Ann. of Math. 128 (1988) 139-151.

[14] Franks J., A variation on the Poincaré-Birkhoff theorem, Contemp. Math. 81 (1988) 139-151.

[15] FRANKS J., Recurrence and fixed points of surface homeomorphims, Ergodic Theory Dynam. Systems 8* (1988) 99-107.

[16] FRANKS J., Area preserving homeomorphisms of open surfaces of genus zero, New York J. Math. 2 (1996) 1-19.

[17] Guillou L., Théorème de translation plane de Brouwer et généralisations du théorème de PoincaréBirkhoff, Topology 33 (1994) 331-351.

[18] Handel M., There are no minimal homeomorphisms of the multipunctured plane, Ergodic Theory Dynam. Systems 12 (1992) 75-83.

[19] Herman M., Sur les courbes invariantes par les difféomorphismes de l'anneau, vol. 2, Astérisque, Soc. Math. de France 144 (1986).

[20] Hocking J., Young G., Topology, Dover Publications, New York, 1961.

[21] Hofer H., ZeHnder E., Symplectic Invariants and Hamiltonian Dynamics, Birkhäuser, 1994.

$4^{\mathrm{e}}$ SÉRIE - TOME $36-2003-\mathrm{N}^{\circ} 1$ 
[22] KÉRÉKJÁRTÓ B.V., Vorlesungen über Topologie (I), Springer-Verlag, Berlin, 1923.

[23] Le CALVEz P., Une propriété dynamique des homéomorphismes du plan au voisinage d'un point fixe d'indice > 1, Topology 38 (1999) 23-35.

[24] Le Calvez P., Yoccoz J.-C., Un théorème d'indice pour les homéomorphismes du plan au voisinage d'un point fixe, Ann. of Math. 146 (1997) 241-293.

[25] Le Calvez P., Yoccoz J.-C., Suite des indices de Lefschetz des itérés pour un domaine de Jordan qui est un bloc isolant, Manuscrit.

[26] Le RouX F., Dynamique des homéomorphismes de surfaces, versions topologiques des théorèmes de la fleur de Leau-Fatou et de la variété stable, Prépublication, Orsay.

[27] MATHER J., Invariant subsets of area-preserving homeomorphisms of surfaces, Adv. Math. Suppl. Stud. 7B (1994) 331-351.

[28] Newman M.H.A., Elements of the Topology of Plane Sets of Points, Cambridge University Press, Cambridge, 1951.

[29] Nikishin N.A., Fixed points of diffeomorphisms of two-dimensional spheres preserving an oriented plane, Funktional Anal. i Prilozen. 8 (1974) 84-85.

[30] PereZ-Marco R., Fixed points and circles maps, Acta Math. 179 (1997) 243-294.

[31] Pelikan S., Slaminka E., A bound for the fixed point index of area-preserving homeomorphisms of two-manifolds, Ergodic Theory Dynam. Systems 7 (1987) 463-479.

[32] Salamon D., Zehnder E., Morse theory for periodic solutions of Hamiltonian systems and the Maslov index, Comm. Pure Appl. Math. 45 (1992) 1303-1360.

[33] SCHWARz M., On the action spectrum for closed symplectically aspherical manifolds, Pacific $J$. Math. 193 (2) (2000) 419-461.

[34] SimON C.P., A bound for the fixed-point index of an area-preserving map with applications to mechanics, Inventiones Math. 26 (1974) 187-200.

\author{
Patrice LE CALVEZ \\ Laboratoire Analyse, \\ Géométrie et Applications, \\ U.M.R. C.N.R.S. 7539, \\ Institut Galilée, \\ Université Paris Nord, \\ 93430 Villetaneuse, France
}

E-mail : lecalvez@math.univ-paris13.fr 